\title{
Possible role of antioxidative capacity of (-)-epigallocatechin-3-gallate treatment in morphological and neurobehavioral recovery after sciatic nerve crush injury
}

\author{
Waleed M. Renno, PhD, ${ }^{1}$ Ludmil Benov, PhD, ${ }^{2}$ and Khalid M. Khan, PhD' \\ Departments of ${ }^{1}$ Anatomy and ${ }^{2}$ Biochemistry, Faculty of Medicine, Kuwait University, Safat, Kuwait
}

OBJECTIVE This study examined the capacity of the major polyphenolic green tea extract (-)-epigallocatechin-3-gallate (EGCG) to suppress oxidative stress and stimulate the recovery and prompt the regeneration of sciatic nerve after crush injury.

METHODS Adult male Wistar rats were randomly assigned to one of 4 groups: 1) Naïve, 2) Sham (sham injury, surgical control group), 3) Crush (sciatic nerve crush injury treated with saline), and 4) Crush+EGCG (sciatic nerve crush injury treated with intraperitoneally administered EGCG, $50 \mathrm{mg} / \mathrm{kg}$ ). All animals were tested for motor and sensory neurobehavioral parameters throughout the study. Sciatic nerve and spinal cord tissues were harvested and processed for morphometric and stereological analysis. For the biochemical assays, the time points were Day 1, Day 7, Day 14, and Day 28 after nerve injury.

RESULTS After sciatic nerve crush injury, the EGCG-treated animals (Crush+EGCG group) showed significantly better recovery of foot position and toe spread and $50 \%$ greater improvement in motor recovery than the saline-treated animals (Crush group). The Crush+EGCG group displayed an early hopping response at the beginning of the 3rd week postinjury. Animals in the Crush+EGCG group also showed a significant reduction in mechanical allodynia and hyperalgesia latencies and significant improvement in recovery from nociception deficits in both heat withdrawal and tail flick withdrawal latencies compared with the Crush group. In both the Crush+EGCG and Crush groups, quantitative evaluation revealed significant morphological evidence of neuroregeneration according to the following parameters: mean crosssectional area of axons, myelin thickness in the sciatic nerve (from Week 4 to Week 8), increase of myelin basic protein concentration and gene expression in both the injured sciatic nerve and spinal cord, and fiber diameter to axon diameter ratio and myelin thickness to axon diameter ratio at Week 2 after sciatic nerve injury. However, the axon area remained much smaller in both the Crush+EGCG and Crush groups compared with the Sham and Naïve groups. The number of axons per unit area was significantly decreased in the Crush+EGCG and Crush groups compared with controls. Sciatic nerve injury produced generalized oxidative stress manifested as a significant increase of isoprostanes in the urine and decrease of the total antioxidant capacity (TAC) of the blood from Day 7 until Day 14. EGCG-treated rats showed significantly less increase of isoprostanes than saline-treated animals and also showed full recovery of TAC levels by Day 14 after nerve injury. In spinal cord tissue analysis, EGCG-treated animals showed induced glutathione reductase and suppressed induction of heme oxygenase 1 gene expression compared with nontreated animals.

CONCLUSIONS EGCG treatment suppressed the crush-induced production of isoprostanes and stimulated the recovery of the TAC and was associated with remarkable alleviation of motor and sensory impairment and significant histomorphological evidence of neuronal regeneration following sciatic nerve crush injury in rats. The findings of this study suggest that EGCG can be used as an adjunctive therapeutic remedy for nerve injury. However, further investigations are needed to establish the antioxidative mechanism involved in the regenerative process after nerve injury. Only upregulation of glutathione reductase supports the idea that EGCG is acting indirectly via induction of enzymes or transcription factors.

https://thejns.org/doi/abs/10.3171/2016.10.SPINE16218

KEY WORDS EGCG; oxidative stress; neuroregeneration; neuroprotection; stereological analysis; rat

ABBREVIATIONS $C_{T}=$ threshold cycle; $C V=$ coefficient of variation; $E G C G=(-)$-epigallocatechin-3-gallate; $E P T=$ extensor postural thrust; $M B P=$ myelin basic protein; RT-PCR = real-time-polymerase chain reaction; TAC = total antioxidant capacity; WRL = withdrawal response latency.

SUBMITTED February 22, 2016. ACCEPTED October 14, 2016.

INCLUDE WHEN CITING Published online August 4, 2017; DOI: 10.3171/2016.10.SPINE16218. 
A STRONG association has been shown between the intake of the major polyphenolic green tea extract (-)-epigallocatechin-3-gallate (EGCG) and vital biological mechanisms that expedite regeneration and neuroprotection and improve cognitive function and learning ability. ${ }^{21,59,68,69}$ It has been suggested that the beneficial effects may result from EGCG's antioxidant activity in reducing intracellular reactive oxygen species and their cascading destructive consequences. ${ }^{23,38,73}$ Another study suggested that EGCG has compelling neuroprotective effects in a D-galactose-induced mouse model of aging through antiapoptotic and antioxidative mechanisms. ${ }^{22}$

Recently, we have shown that EGCG exerted a neuroprotective effect on the spinal cord by modulating neurological function when intravenously infused into adult rats in both acute and chronic spinal cord injury models. ${ }^{48,52}$ Moreover, EGCG attenuated peripheral nerve degeneration of rat sciatic nerve crush injury, an effect that seemed to be associated with its antiapoptotic properties. ${ }^{49,50}$ Another characteristic of EGCG reported by many investigators is its antiinflammatory activity. ${ }^{57,58,72}$ EGCG pretreatment abolished the cytokine-mediated upregulation of inducible nitric oxide synthase (iNOS) and intercellular adhesion molecule-1 (ICAM-1) via the JAK/STAT cascade. Via an antiinflammatory mechanism, EGCG has the capacity of protecting against various neurodegenerative diseases, and it has been shown to prevent TNF$\alpha$-induced $\mathrm{NF}-\kappa \mathrm{B}$ activation, thereby upregulating the ATP-binding membrane cassette transporter A1 (ABCA1) via the Nrf2/Keap1 pathway in macrophage foam cells. ${ }^{28}$ EGCG acts against infrasound-induced neuronal impairment by inhibiting microglia-mediated inflammation through a potential $\mathrm{NF}-\kappa \mathrm{B}$ pathway-related mechanism in the CNS. ${ }^{6}$ However, in vivo mechanisms of EGCG with respect to the amelioration of neurobehavioral parameters resulting from peripheral nerve injury are not fully understood. Moreover, no thorough morphometric analysis of the effect of EGCG treatment on the sciatic nerve injury throughout the recovery period (Weeks 2-8) has been previously reported.

In this study, we examined the possible antioxidative and/or antiinflammatory role of EGCG associated with beneficial histopathological changes at different time points following sciatic nerve crush injury. Furthermore, we analyzed the relationship between sensory and motor neurobehavioral parameters and quantitative morphological changes in individual nerve fibers (axon plus myelin sheath), such as fiber diameter, axon diameter, myelin thickness, and g-ratio, during regeneration of the peripheral nerve.

\section{Methods \\ Animals}

Male Wistar rats (weight 250-300 g) were obtained from the Animal Resource Center of the Kuwait University Health Sciences Centre. The animals were kept under conditions of constant temperature $\left(23^{\circ} \mathrm{C} \pm 2^{\circ} \mathrm{C}\right)$ and humidity, with a 12-hour light/dark cycle. The rats were housed in pairs and were provided with food and water ad libitum. Approval was obtained for all procedures from the Animal Ethics Committee of the Kuwait University Health Sciences Center, and the study was carried out in accordance with the US guidelines. All efforts were made to minimize the number of animals used in the study and their suffering.

A total of 204 rats were used in this study. The study consisted of 3 separate experiments (neurobehavioral, morphometric, and biochemical). For each experiment, equal numbers of animals were randomly assigned to the following 4 treatment groups: 1) Naïve (no surgery or sciatic nerve injury); 2) Sham (sham-injury surgical control group); 3) Crush (saline-treated, crushed sciatic nerve); or Crush+EGCG (50 mg/kg EGCG-treated, crushed sciatic nerve).

Sixty rats were used in the neurobehavioral experiment (15 per group); all of these rats were killed at the end of the experiment (Week 10). Forty-eight rats were used in the morphometric experiment (12 per group), with 3 rats in each group being killed at 2, 4, 6, and 8 weeks. Ninety-six rats were used in the oxidative pathway biochemical experiment ( 24 per group), with 6 rats per group being killed at each of the following time points: Day 1, Day 7, Day 14, and Day 28.

\section{Procedures for Control and Surgical Groups}

The Naiive group received no surgery or injury. The Sham group was subjected to all the surgical steps (cutting the skin, separating muscle and exposing the sciatic nerve) except crushing the nerve. In the Crush and Crush+EGCG groups, the sciatic nerve crush injury was performed as previously described. ${ }^{50-52}$ Briefly, animals were anesthetized with an intraperitoneal injection of a mixture of ketamine and xylazine. The sciatic nerve was crushed at a distance of $10 \mathrm{~mm}$ from the sciatic notch for 60 seconds using micro mosquito forceps $(12.5 \mathrm{~cm}$, straight, Item No. 500451, [09F S/N], World Precision Instruments, Inc.). The nerve was examined to ensure that the epineurial sheath was intact but translucent (axotomy). Then it was replaced under the muscle, and the skin incision was sutured. ${ }^{5,6,39,50-53,56,70}$ Animals in the Crush+EGCG group were injected intraperitoneally with $50 \mathrm{mg} / \mathrm{kg}$ EGCG (Sigma-Aldrich, product no. E4143, dissolved in saline) daily for 3 days starting 1 hour after the sciatic nerve crush injury. ${ }^{12,34,48,52,62,64}$ The stock solution was made fresh daily, using $50 \mathrm{mg}$ EGCG and $1 \mathrm{ml} \mathrm{sa-}$ line. Animals in the Crush group were administered an equal volume of saline according to the same schedule. Numerous previously described measures were followed in order to minimize animal-animal variation in producing the injury and to induce a standard direct trauma. ${ }^{20,40 \text {, }}$ 49-53,56,70,67,71 The crush-lesioned rats were randomly distributed among the experimental groups to minimize group variations as much as possible.

\section{Assessment of Functional Recovery}

All animals were evaluated for walking track analysis preoperatively. All surviving animal groups (Naïve, Sham, Crush+Saline, and Crush+EGCG) were evaluated once a week following surgery (zero point) as described ear- 
lier. ${ }^{49-53}$ All the tests were repeated 3 times (at 3-minute intervals), and the average of the 3 readings was used for data analysis. Investigators were blinded to all treatments in all experiments. Several tests of reflexive sciatic nerve function (foot position, toe spread, extensor postural thrust [EPT] and hopping tests) were conducted as described in our previous studies. ${ }^{49,53}$ Briefly, EPT was measured by calculating the functional deficit in this feature; thus higher values indicate greater deficit. ${ }^{37}$ The hopping test was done to test several integrated functions. Each leg of the rat was suspended above a table surface, and then the leg was placed on the table and was slowly moved laterally, with one foot touching the table surface at a time. The scoring was based on whether the rat hopped on the foot that was touching the table surface ( 1 for hopping, 0 for no hopping). ${ }^{63}$

Four different neurobehavioral sensory tests were implemented as follows.

1. Tactile allodynia: The von Frey method was used to evaluate the sensitivity of the skin to tactile stimulation as described previously. ${ }^{49,50,51}$ Mechanical allodynia thresholds from the right and left hind limbs were measured in different animal groups and then the withdrawal reflex latencies (mean \pm SD) were calculated.

2. Mechanical hyperalgesia: Paw pressure thresholds expressed in grams were measured using an analgesy meter (Model 37215, UGO Basile). Three consecutive mechanical nociceptive threshold values were measured for each hind limb, and the mean and standard deviation (SD) of the paw pressure latencies were calculated. ${ }^{13,37,48-50,53}$

3. Thermal nociception: A modified hot-plate test $\left(50^{\circ} \mathrm{C}\right.$, Model 39D Hot Plate Analgesia Meter, Columbus Instruments) was performed, with the withdrawal response threshold defined as the time elapsed from the onset of hot-plate contact to the withdrawal of the hind paw (paw flick), recorded to the nearest 0.1 second; a standard cutoff latency of 20 seconds was employed to prevent tissue damage. ${ }^{32} \mathrm{~A}$ normal response is considered withdrawal within 4 seconds. The withdrawal response threshold was measured for both hind limbs. Each hind limb was tested 3 times, with 20 minutes between tests to avoid sensitization. The withdrawal response latency (WRL) was calculated, and the $3 \mathrm{WRL}$ values were averaged to obtain a single value for each animal.

4. Tail flick: A UGO Basile tail flick unit (Model 7360) was used to assess spinally mediated nociceptive thresholds, recording the amount of time that it took for an animal to move its tail away from the heat ${ }^{36} \mathrm{~A}$ cutoff time of 20 seconds was used to prevent tissue damage. ${ }^{32,49,50}$

\section{Statistical Analysis}

All behavioral data were analyzed by 2-way repeated measures ANOVA followed by Bonferroni's F-test and Fisher least significant difference (LSD) comparison post hoc test or Student t-test, as appropriate. All statistical analysis was performed using SPSS (version 21.0, IBM Corp.); $\mathrm{p}$ values less than 0.05 were considered statistically significant.

\section{Morphometric and Stereological Analyses}

Tissue Processing for Morphometric Analysis

At the end of Week 2, Week 4, Week 6, and Week 8, 3 animals from each experimental group were randomly selected for histopathological evaluation as previously described. ${ }^{7,850}$ Briefly, animals were killed by decapitation, and the right sciatic nerve from each animal was exposed and removed. The specimen was then fixed by immersion in a mixture of $2 \%$ paraformaldehyde and $2 \%$ glutaral-

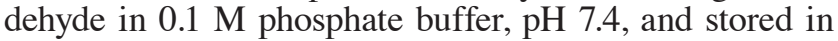
the fixative overnight at $4^{\circ} \mathrm{C}$. On the following day, the specimens were rinsed in phosphate buffer ( $\mathrm{pH}$ 7.4) twice, postfixed in $1 \%$ osmium tetroxide, dehydrated through a graded alcohol series, immersed in propylene oxide, and embedded in Epon resin (Hexion). ${ }^{50}$ Semi-thin cross sections $(1 \mu \mathrm{m})$ were cut from each block using an RMC MT-7 ultramicrotome (Research and Manufacturing Co.) and stained with $1 \%$ toluidine blue for histopathological examination and morphometric analysis. ${ }^{50}$

\section{Quantification of Myelinated Fibers}

Sciatic nerves removed from rats at 2, 4, 6, and 8 weeks postinjury were processed for stereological and histopathological examination. Stereological analyses of these sciatic nerves were performed by an experimenter blinded to the groups according to principles described previously. $3,17,26,67$ A stereological workstation composed of a digital camera (Nikon COOLPIX 4500), a manual dial indicator-controlled specimen stage, ${ }^{29}$ and a light microscope (Olympus DP71 optical microscope) were used for counting the axons. Sections from each nerve (at least 5 sections from each rat) were examined in a systematic, uniform, and random manner, and nerve fibers were counted (Fig. 1A). Area sampling of nerve sections was done with 100 $\times 100-\mu \mathrm{m}$ successive, systemic random steps along adjacent areas of each nerve sample, and representative fields were photographed under a $100 \times$ oil-immersion objective lens (total magnification 1000x). At least 10 photos per sample were taken randomly at $1000 \times$ for morphometric analysis. Digital images were captured with an Olympus DP71 digital camera mounted on top of the microscope ${ }^{65}$ All morphometric parameters were calculated by light microscopy using Image-Pro Plus version 6.0.0.260 for Windows 2000/XP Professional (Media Cybernetics).$^{14}$ Figure 1A shows a representative photograph of a cross section of sciatic nerve myelinated axons stained with toluidine blue. The unbiased counting frame method (square sample area $900 \mu^{2}$ ) was used to obtain an estimate of total axon number from nerve cross sections. A counting frame was placed on a monitor, and the sampled area was selected in a systematic, uniform, and random manner. ${ }^{29} \mathrm{~A}$ mean sampling of each sectioned nerve profile was done in a 70 $\times 70-\mathrm{mm}$ frame size in a systematic random manner. This ensured that all locations within a nerve cross section (1$\mu \mathrm{m}$ thickness) were equally represented and that all axon profiles were sampled with equal probability regardless of shape, size, orientation, or location. $18,19,25,41,43,44,65,71$

Counting of axons was performed using a $100 \times$ oil objective lens (total magnification 1000x), which allowed accurate recognition of myelinated nerve fibers. The camera was connected to a $500-\mathrm{MHz}$ Pentium-III personal 

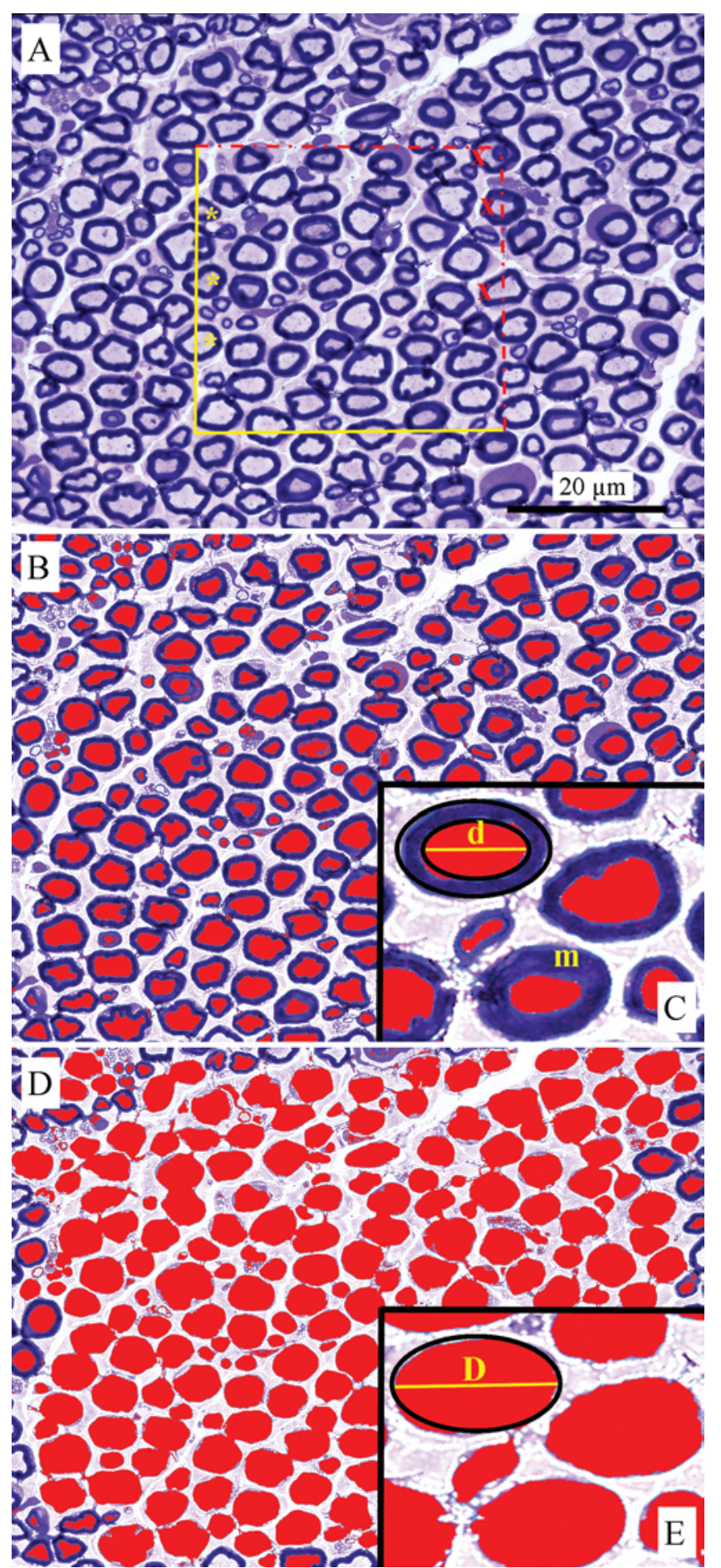

computer. The 3 color channels of the camera were balanced by adjusting the light intensity and camera gain and offset, using Olympus software. Images were viewed on a 15-inch Samsung SyncMaster 500S color monitor (Fig. 1B-E). The axon diameter (d) and the nerve fiber diameter (D) were measured automatically using Image-Pro Plus 6.0 image analysis software and were used to calculate myelin thickness $(m=[D-d] / 2)$ and g-ratio $(d / D)$. The thickness measurements acquired from all sampled axons
FIG. 1. A: Representative photomicrograph of a cross section of sciatic nerve myelinated axons stained with toluidine blue. The unbiased counting frame method with a square sample area was used to count the myelinated axons. The nerve profiles marked with yellow asterisks along the solid yellow lines (inclusion lines) were included in the counts, but the profiles marked with red Xs touching the broken red lines (exclusion lines) were excluded. B-E: Representative photomicrographs of a cross section of sciatic nerve myelinated axons stained with toluidine blue demonstrating the method of calculating myelin thickness and $g$-ratios. For calculating the myelin thickness $(m=[D-d] / 2)$ and $g$ ratio $(d / D)$, the axon diameter (d) and the nerve fiber diameter (D) were measured automatically using Image-Pro Plus 6.0. Thickness measurements from all sampled axons were averaged to obtain the mean myelin thickness. Magnifications: B and D, $1000 \times(100 \times$ oil immersion $\times 10 \times$ ocular); $C$ and $E, 3000 x$. Figure is available in color online only.

were then averaged to obtain the mean myelin thickness. Myelin sheath thickness was calculated as the difference between the fiber diameter and the axon diameter, divided by 2: $(D-d) / 2$. The myelin thickness/axon diameter ratio was calculated as $(D-d) / 2 d$, and the nerve fiber diameter/ axon diameter ratio was $\mathrm{D} / \mathrm{d}$.

The number of axons in the image $(\mathrm{N})$ was calculated by using the following equation: $\mathrm{N}=(\mathrm{A} \times \mathrm{n}) / \mathrm{a}$, where $\mathrm{n}$ is the number of axons in small square $n$, which is identified through Image-Pro Plus; a is the area of the small square, and $\mathrm{A}$ is the total area of the image (length $\times$ width). Also, the g-ratio was calculated as the quotient obtained by dividing the axon diameter by the fiber diameter, and the coefficient of variation (CV) was calculated as (standard deviation $/$ mean) $\times 100$. ANOVA multicomparison test was used, followed by Bonferroni post hoc analysis.

\section{Morphometric Data Analysis}

Images of the distal and proximal portions of the right sciatic nerve obtained from 9 rats per group were captured and digitized using Image-Pro Plus software. The distal and proximal portions of the right sciatic nerves were analyzed separately; the average number of the fibers analyzed per nerve segment was 155 . A total of 790 images (10 from each section) were digitized and stored in uncompressed tagged image file format (TIFF) with 24-bit RGB class and $640 \times 480$-pixel resolution. All data are presented as the mean \pm SD. All calculations and all statistical procedures were performed using SPSS (version 21.0). The group values were compared using a Mann-Whitney U-test. A p value of less than 0.05 was considered significant.

\section{Biochemical Analyses \\ Oxidative Stress Measurements}

A total of 24 rats (6 from each of the 4 groups) were used for each time point (1, 7, 14, and 28 days). Sciatic nerve, spinal cord, blood, and urine samples were harvested at each time point and stored at $-80^{\circ} \mathrm{C}$ until analyses were performed. The following assays were performed: 1) total antioxidant capacity in blood (Antioxidant Assay Kit, No. 709001, Cayman Chemical); 2) urine isoprostanes (8-Isoprostane EIA Kit, No. 516351, Cayman Chemical); 3) lipid peroxidation (Lipid Hydroperoxide LPO Assay Kit, No. 705003, Cayman Chemical); and 4) glutathione to 
TABLE 1. Summary of gene information for oxidation and inflammation pathways

\begin{tabular}{|c|c|c|c|}
\hline Gene Symbol & Gene Name & Accession No. & Life Technologies Gene Assay ID \\
\hline Tank & TRAF family member-associated NFKB activator & NM_001164073.1 & Rn00595794_m1 \\
\hline $\operatorname{Tnf}{ }^{*}$ & Tumor necrosis factor & NM_012675.3 & Rn01525859_g1 \\
\hline$\| 1 / b$ & Interleukin 1 beta & NM_031512.2 & Rn00580432_m1 \\
\hline Ptgs $2 \dagger$ & Prostaglandin-endoperoxide synthase 2 & NM_017232.3 & Rn01483828_m1 \\
\hline Cyp1a1 & Cytochrome P450, family 1 , subfamily a, polypeptide 1 & NM_012540.2 & Rn01418021_g1 \\
\hline Hmox1 & Heme oxygenase 1 & NM_012580.2 & Rn01536933_m1 \\
\hline Nr3c1 $\ddagger$ & Nuclear receptor subfamily 3 , group C, member 1 & NM_012576.2 & Rn00561369_m1 \\
\hline Gabpa§ & GA binding protein transcription factor, alpha subunit & NM_001108841.1 & Rn01767215_m1 \\
\hline \multicolumn{4}{|c|}{$\begin{array}{l}\text { ID = identifier. } \\
\text { All genes listed are Rattus norvegicus (Norway rat) genes. } \\
\text { * Also known as Tnfa, RATTNF, and TNF-alpha. } \\
\dagger \text { Also known as Cox2. } \\
\text { ‡ Also known as GR (glucocorticoid receptor). } \\
\S \text { Also known as Nrf2a. }\end{array}$} \\
\hline
\end{tabular}

glutathione disulfide ratio (GSH/GSSG-412, Catalog No. 21040, OxisResearch). All tests were performed according to manufacturers' protocols.

\section{Real-Time-Polymerase Chain Reaction Measurements}

Total RNA Isolation and Reverse Transcription. To perform real-time-polymerase chain reaction (RT-PCR) measurements, we isolated total RNA from frozen nerve and spinal cord samples using Trizol reagent (Life Technologies Inc.). RNA quantities were evaluated using multivolume analysis of nucleic acids at $260-\mathrm{nm}$ absorption spectroscopy (Epoch Multi-Volume Spectrophotometer System, BioTek Instruments, Inc.). Complementary DNA (cDNA) was synthesized by random priming of $2-5 \mu \mathrm{g}$ total RNA using a High Capacity cDNA Reverse Transcription Kit (Applied Biosystems) at $85^{\circ} \mathrm{C}$ for 2 hours and stored at $-20^{\circ} \mathrm{C}$.

For RT-PCR, optimized TaqMan probe/primers kits used for the detection of specific genes and $\beta$-actin (housekeeping gene) were purchased from Applied Biosystems. The complete information on the specific genes used in this study is listed in Table 1. RT-PCR was performed in duplicates using the Applied Biosystems 7500 PCR system. $1,24,25,44,50,52,61$ Amplification of the above genes was done in 96-well reaction plates. The reaction mixture contained $3 \mu \mathrm{l}$ of cDNA, $200 \mathrm{nmol} / \mathrm{L}$ of TaqMan probe/primer assays, and $12.5 \mu \mathrm{l}$ of qPCR master mix (Applied Biosystems) in each well. A nontemplate PCR-negative control and a positive PCR control were included. Quantitative mRNA expression was determined by the comparative threshold cycle $\left(\mathrm{C}_{\mathrm{T}}\right)$ method. The target gene-specific signal was normalized based on the expression levels of the target gene versus reference gene ( $\beta$-actin signal) using the formula $2-\Delta \Delta \mathrm{C}_{\mathrm{T}}=2-\left(\mathrm{C}_{\mathrm{T}}, \beta\right.$ actin $-\mathrm{C}_{\mathrm{T}}$, target gene), resulting in the evaluation of the samples as an n-fold difference relative to that of $\beta$-actin mRNA expression.

\section{RT-PCR Statistical Analysis}

All biochemical RT-PCR data were analyzed by 1-way repeated measures ANOVA followed by the NeumanKeuls test, using SPSS (version 21). Results were considered significant at $\mathrm{p}<0.05$. Data are presented as mean \pm standard error of the mean (SEM).

\section{Results \\ General Assessment}

The hind paws of the Sham animals clinically looked normal following sham surgery. In addition, the skin and muscle wounds did not appear to hinder the animals' gait throughout the progression of the experiment. The toes and foot of the hind limb in the sciatic nerve crush-injured rats without EGCG treatment were intensely flexed. During the first week, the animals were also unable to stand on their operated hind limb. Partial weight bearing was noticed in the crush-injured animals starting from the second week. In contrast, in the EGCG-treated animals the clinical picture and weight bearing were improved over the subsequent weeks compared with the controls.

\section{Behavioral Tests}

The mean values for foot position (Fig. 2A) and toe spread (Fig. 2B) were consistently higher in the Crush+EGCG group than in the Crush, Sham, and Naive groups starting from the end of Week 1 until the end of Week 8 following injury. This difference in progressive improvement of the Crush+EGCG group compared with the other groups was statistically significant $(p<$ 0.001 ). The evaluation of the functional recovery as measured using the extensor postural thrust (EPT) following crush injury and EGCG treatment is shown in Fig. 2C. Crush+EGCG rats displayed more than 50\% improvement in motor recovery $(\mathrm{p}<0.001)$ compared with the Crush group from Week 1 to the end of the experiment at Week 10 after sciatic nerve injury. Similarly, the results of the hopping test (Fig. 2D), which examines several integrated sensory and motor functions, demonstrated significant $(\mathrm{p}$ $<0.0001$ ) improvement in the Crush+EGCG group compared with the Crush group. The Crush+EGCG group dis- 

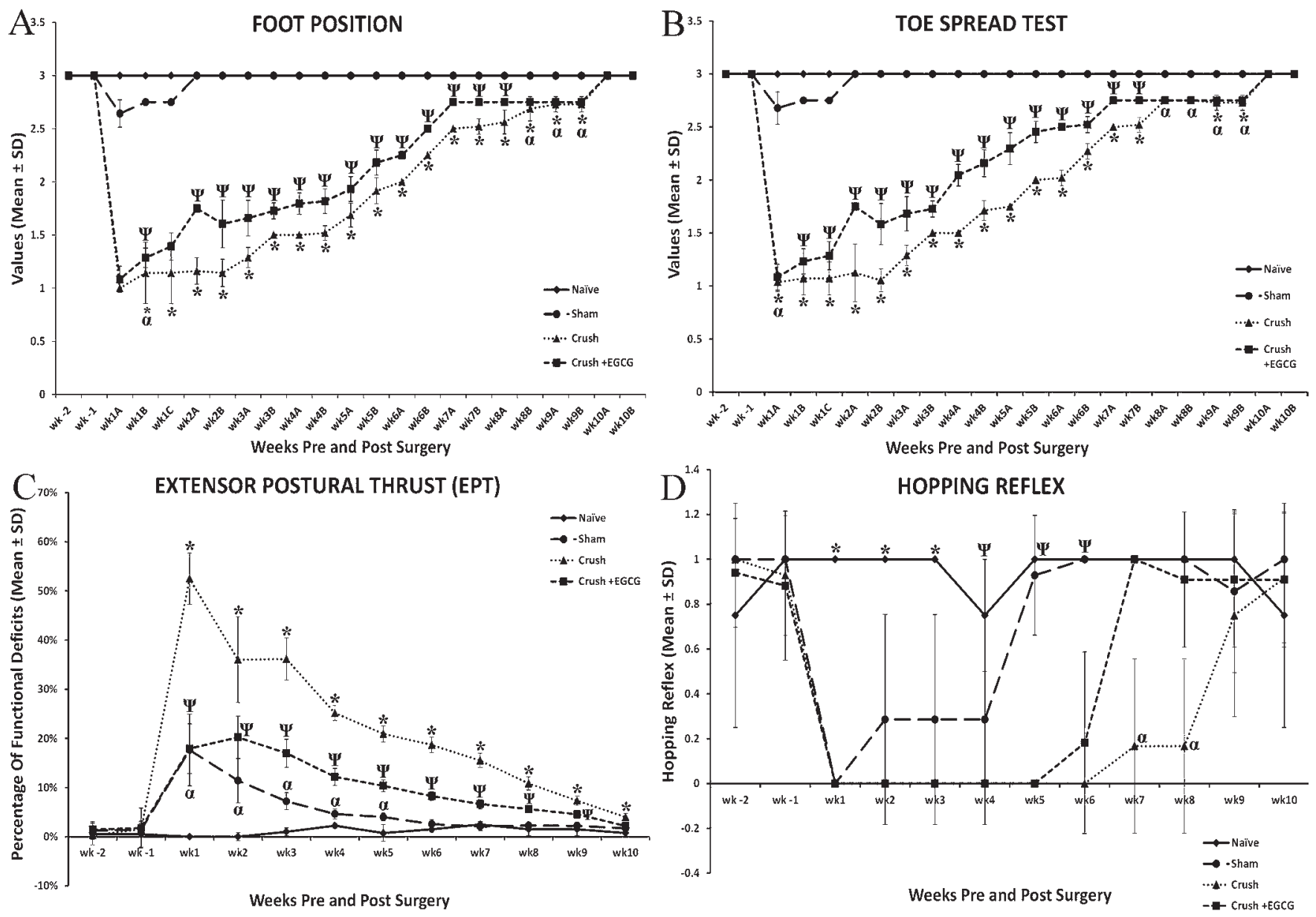

FIG. 2. A: Differences in the mean values for the foot position analysis for the Naïve, Sham, Crush, and Crush+EGCG groups. $\Psi$ indicates $p<0.001$, Crush+EGCG vs Crush, Sham, and Naïve; * indicates $p<0.001$, Crush vs Naïve, Sham, and Crush+EGCG; $\alpha$ indicates no significant difference between Crush+EGCG and Crush. B: Differences in the mean values for the analysis of toe spread outcome. The Crush+EGCG rats showed statistically significant clinical recovery in toe spread following sciatic nerve injury. As with foot position, recovery of toe spread started at the end of postinjury Week 1 and continued until the end of Week 8. $\Psi$ indicates $p<0.001$, Crush+EGCG vs Crush, Sham, and Naïve; * indicates $p<0.001$, Crush vs Sham, Naïve, and Crush+EGCG; $\alpha$ indicates no significant difference between Crush+EGCG and Crush groups. C: Evaluation of functional recovery as measured by EPT following crush injury and EGCG treatment. EPT was measured by calculating the percentage of the functional deficit; thus, a high value indicates a poor outcome. * indicates $p<0.001$, Crush vs Sham, Naïve, and Crush+EGCG; $\Psi$ indicates $p<$ 0.001 , Crush+EGCG vs Crush, Sham, and Naïve; no significant difference between Crush+EGCG and Sham; $\alpha$ indicates $p<0.01$, Sham vs Naïve group. D: Differences in the mean values of the hopping test outcome among the experimental animal groups. The Crush+EGCG group displayed an early hopping response (as calculated by the percentage of recovered animals) in the beginning of Week 7 postsurgery. * indicates $p<0.001$, Naïve vs Sham, Crush, and Crush+EGCG; $\Psi$ indicates $p<0.001$, Naïve vs Crush and Crush+EGCG; $\alpha$ indicates $p<0.0001$, Crush vs Crush+EGCG, Sham, and Naïve. Values are expressed as means and $\mathrm{SDs}$ (error bars). $\mathrm{A}=$ first reading of the week; $\mathrm{B}=$ second reading; $\mathrm{C}=$ third reading.

played an early recovery of the hopping response in the beginning of Week 7 postsurgery (Fig. 2D).

Several neurobehavioral sensory tests were performed, including assessment of allodynia, mechanical hyperalgesia, thermal hyperalgesia, and tail flick latencies. The time course graph displaying the assessment of allodynia time latency of the different experimental groups before and after sciatic nerve injury is shown in Fig. 3A. The Crush+EGCG group showed a significant $(p<0.001)$ reduction in withdrawal reflex latency time compared with the Crush group from the beginning of Week 2 to the end of the experiment at Week 10. Moreover, it was noticed that the latency times of the Crush+EGCG animals followed that of the Sham group. Likewise, in the paw pressure latency (mechanical hyperalgesia) test, Crush+EGCG rats showed a significant $(\mathrm{p}<0.001)$ decrease in paw withdrawal reflex latency periods compared with Crush group starting from Week 3 (Fig. 3B). This decrease lasted up until the end of the experiment at Week 10. Nerve crush injury produced a severe nociception deficit in the Crush group as demonstrated using the hot plate analgesia meter (Fig. 3C). On the other hand, the Crush+EGCG group showed a significant $(\mathrm{p}<0.001)$ nociceptive recovery starting at Week 2 , which was maintained throughout until the end of the experiment at Week 10 (Fig. 

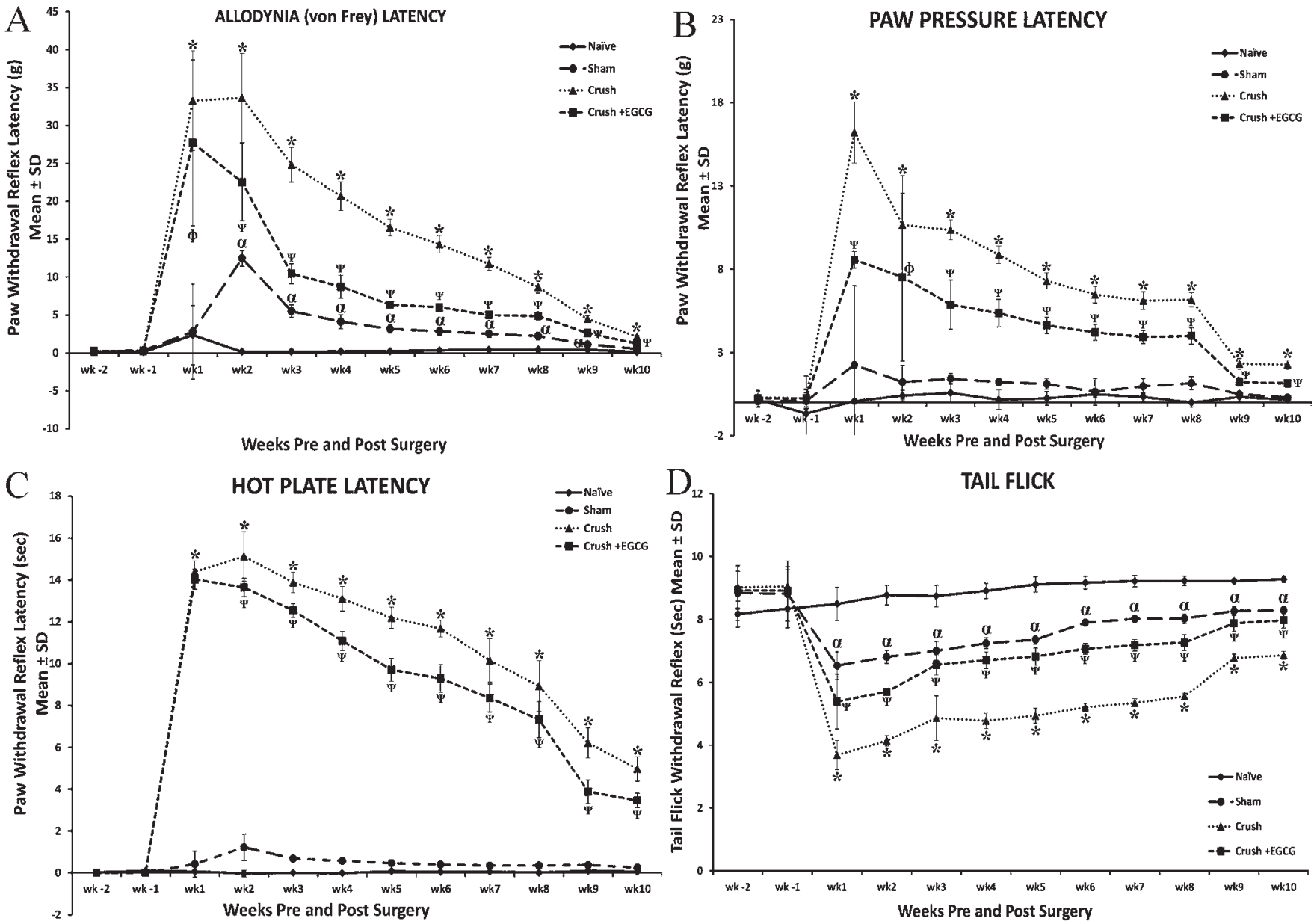

FIG. 3. A: Time course of tactile allodynia. The Crush+EGCG group showed a significant reduction in withdrawal reflex latency time compared with the Crush group from the beginning of Week 2 until the end of the experiment at Week 10. Moreover, the latency times of the Crush+EGCG animals followed those of the Sham group. * indicates $p<0.001$, Crush vs Crush+EGCG, Sham, and Naïve; $\Psi$ indicates $p<0.001$, Crush+EGCG vs Crush, Sham, and Naïve; $\alpha$ indicates $p<0.001$, Sham vs Crush, Crush+EGCG, and Naïve; $\Phi$ indicates $p<0.001$, Crush+EGCG vs Sham and Naïve. Values are expressed as means and SDs (error bars). B: Time course of paw pressure latency (mechanical hyperalgesia). The Crush+EGCG group showed a significant decrease in paw withdrawal reflex latency periods compared with the Crush group starting from Week 3 and continuing until the end of the experiment (Week 10). (Note that the paw withdrawal latency rather than the absolute withdrawal reflex was measured, so we expect the values to be higher in the Crush group compared with the controls.) * indicates $p<0.001$, Crush vs Crush+EGCG, Sham, and Naïve; $\Psi$ indicates $p<0.001$, Crush+EGCG vs Crush, Sham, and Naïve groups; $\Phi$ indicates $p<0.002$, Crush+EGCG animals compared with Naïve and Sham groups. Values are expressed as means and SDs (error bars). C: Time course of hot plate latency. Nerve crush injury produced a severe nociception deficit in the Crush group. The Crush+EGCG group showed a significant nociceptive recovery staring at Week 2 and maintained until the end of the experiment (Week 10). * indicates $p<0.001$, Crush vs Crush+EGCG, Sham, and Naïve; $\Psi$ indicates $p<0.001$, Crush+EGCG vs Crush, Sham, and Naïve. Values are expressed as means and SDs (error bars). D: Time course of tail flick withdrawal latency. The Crush+EGCG group showed significant nociceptive recovery compared with Crush (saline-treated) group by the end of the 2nd week postsurgery. This nociception improvement was sustained until the end of the experiment (Week 10). Furthermore, the EGCG-treated group displayed a significant decrease in nociception compared with the Sham and Naïve groups. ${ }^{*}$ indicates $p<0.001$, Crush vs Crush+EGCG, Sham, and Naïve; $\Psi$ indicates $p<0.001$, Crush+EGCG vs Crush, Sham, and Naïve; $\alpha$ indicates $p<0.001$, Sham vs Crush+EGCG, Crush, and Naïve. Values are expressed as means and SDs (error bars).

3C). The tail flick withdrawal latency in the Naïve, Sham, Crush, and Crush+EGCG groups was also examined, and the results are depicted in Fig. 3D. The Crush+EGCG group showed significant $(\mathrm{p}<0.001)$ nociceptive recovery compared with the saline-treated rats (Crush group) by the end of Week 2 postsurgery. This central nociception improvement was sustained until the end of the experiment at
Week 10. Furthermore, EGCG-treated animals displayed a significant $(\mathrm{p}<0.001)$ decrease in their nociception parameter compared with Sham and Naïve groups (Fig. 3D).

\section{Histological and Morphometric Analysis}

Light Microscopy

Histological examination of the photomicrographs of 
semi-thin transverse sections of sciatic nerves sampled from animals in the Sham group at different time points revealed a normal sciatic nerve appearance. Specimens from these animals showed the regular distribution of small- and large-diameter nerve fibers and normal proportion between myelin sheath thickness, fiber diameter, and intraneural blood vessels (Fig. 4A, D, G, and J). Wallerian degeneration and unmyelinated fibers were evident in the tissue sections of the sciatic nerve $10 \mathrm{~mm}$ distal to the lesion site of animals in the Crush group (Fig. 4B, E, $\mathrm{H}$, and $\mathrm{K}$ ) starting from Week 2 (Fig. 4B) until Week 8 (Fig. 4K) after crush nerve injury. In contrast, nerves of Crush+EGCG (Fig. 4C, F, I, and L) animals showed a remarkable axonal and myelin regeneration and contained a greater number of large newly regenerated nerve fibers that were surrounded by much more myelin compared with the Crush group. At Week 4, Crush+EGCG (Fig. 4F) animals showed remarkable histological improvement in comparison with Crush (Fig. 4E) animals. Crush+EGCG (Fig. 4L) nerves reached almost normal morphology by the end of Week 8 postsurgery in contrast to Crush group rats (Fig. 4K). The histological appearance of nerve specimens from the Crush+EGCG group (Fig. 4L) was similar to that of specimens from the Sham (Fig. 4J) group after 8 weeks postsurgery.

\section{Stereological Analysis}

Morphometric analysis data of sciatic nerves after 2, 4, 6, and 8 weeks postsurgery are presented in Figs. 5, 6, and 7. Figure 5A shows the mean total number of myelinated axons that were calculated for each experimental group at Weeks 2, 4, 6, and 8 following sciatic nerve injuries using a square counting frame. At Week 2 after sciatic injury, the Crush+EGCG and Crush animals showed a significant $(p<0.0001)$ decrease in the mean total myelinated axons compared with Naïve and Sham groups. Starting at Week 4, the mean total number of myelinated axons in the Crush+EGCG and Crush animals increased to the same levels as Naïve and Sham groups and became significantly $(\mathrm{p}<0.0001)$ higher at Week 6 compared with Naïve and Sham control groups. EGCG treatment significantly decreased the number of myelinated axonal fibers in sciatic nerve crush animals compared with Crush group ( $\mathrm{p}$ $<0.0001)$ at Week 6 and Week 8; however, it remained significantly higher $(\mathrm{p}<0.0001)$ compared with Sham and Naïve control groups (Fig. 5A).

The result of the mean cross-sectional areas of myelinated axons obtained from 10 different and randomly selected samples from each animal are depicted in Fig. $5 \mathrm{~B}$. The averaged areas of myelinated axons measured in square micrometers $\left(\mu \mathrm{m}^{2}\right)$ were calculated for each experimental group at Weeks 2, 4, 6, and 8 following sciatic nerve injuries. The Crush and Crush+EGCG rats showed significant $(p<0.0001)$ decrease in the average areas of myelinated axons compared with Sham and Naïve control groups throughout the experimental period. However, the Crush+EGCG animals showed a significant gradual increase in the mean areas of the myelinated axons at Week 6 ( $p<0.03)$, and Week $8(\mathrm{p}<0.0001)$ compared with Crush groups following nerve injury (Fig. 5B).

Myelinated axon diameters (d) (Fig. 6A), myelinated nerve fiber diameter (D) (Fig. 6B), and myelin thickness (Fig. 6C) were stereologically estimated from 10 different random samples. These samples were selected from each animal for each experimental group at Weeks 2, 4, 6, and 8 following sciatic nerve injuries using a square counting frame. The Crush and Crush+EGCG groups displayed significant $(\mathrm{p}<0.0001)$ decrease in axon diameter size at all the time points assessed following nerve injury compared with Sham and Naïve animals. However, Crush+EGCG groups showed no significant change compared with Crush animals even at Week 8 after nerve injury. Similarly, the Crush and Crush+EGCG groups displayed a significant $(\mathrm{p}<0.0001)$ decrease in nerve fiber diameter sizes at all the time points assessed following nerve injury compared with Sham and Naïve animals. However, the Crush+EGCG group showed significant $(\mathrm{p}<0.008)$ nerve fiber diameter increase by the end of Week 8 postinjury (Fig. 6B). The calculated myelin thickness for the Crush and Crush+EGCG groups at different time points following sciatic nerve injury showed a significant decrease $(\mathrm{p}<$ 0.0001) throughout the experiment starting from Week 2 until Week 8 (Fig. 6C). However, the myelin thickness in Crush+EGCG animals started to increase significantly at Week $6(\mathrm{p}<0.05)$ and became much greater at Week 8 $(p<0.0001)$ compared with values obtained in the Crush group.

Stereological analysis of the g-ratios described as the axon diameter to myelinated fiber diameter ratio (Fig. 7A) and myelin thickness to axon diameter ratio (Fig. 7B) were calculated from the data measurements obtained from each animal. The Crush and Crush+EGCG groups showed a significant $(\mathrm{p}<0.001)$ increase in the axon diameter to myelinated fiber diameter ratio at Week 2 and Week 4 postinjury. The Crush+EGCG animals displayed significantly $(\mathrm{p}<0.05)$ lower axon diameter to myelinated fiber diameter ratio compared with Crush group only at Week 2 following injury (Fig. 7A). The Crush and Crush+EGCG groups showed a significant $(\mathrm{p}<0.0001)$ decrease in the myelin thickness to axon diameter ratio at Week 2 and Week 4 compared with all other groups (Fig. 7B). Conversely, the Crush+EGCG animals exhibited significantly $(p<0.01)$ higher myelin thickness to axon diameter ratio throughout at Week 6 and Week 8 following injuries compared with Crush group (Fig. 7B).

In support of the stereological data analysis, the myelin basic protein (MBP) concentration and gene expression analyses of the sciatic nerve and spinal cord samples showed a similar pattern at different time points following nerve injury (Fig. 8). EGCG treatment significantly ( $\mathrm{p}<$ 0.002) increased the MBP concentration in the sciatic nerve samples starting from Day 1 until Day 14 postinjury compared with Crush group (Fig. 8A and B). RT-PCR analysis of MBP from sciatic nerve tissues revealed a gradual increase in MBP gene expression at Day 3 and Day 7 following EGCG treatment. At Day 14, there was significant (p $<0.01$ ) increase in the MBP gene expression in the sciatic nerves of the EGCG-treated group compared with Crush animals (Fig. 8C). Similarly, analysis of MBP concentration from the spinal cords at different time points following nerve injury showed a significant $(p<0.005)$ increase in the MBP concentration of the EGCG-treated animals at 




FIG. 4. Photomicrographs of semi-thin transverse sections of sciatic nerves obtained from animals in the Sham (A, D, G, and J), Crush (B, E, H, and K), and Crush+EGCG (C, F, I, and L) groups at different time points. Sciatic nerves from Crush and Crush+EGCG animals show the presence of smaller minifascicle nerve fibers as time progressed following crush injury. Nonetheless, sciatic nerve sections of Crush+EGCG $(C, F, I$, and $L)$ animals showed remarkable nerve regenerations compared with those from the Crush $(\mathrm{B}, \mathrm{E}, \mathrm{H}$, and $\mathrm{K})$ group starting from Week 2 . Figure is available in color online only. 

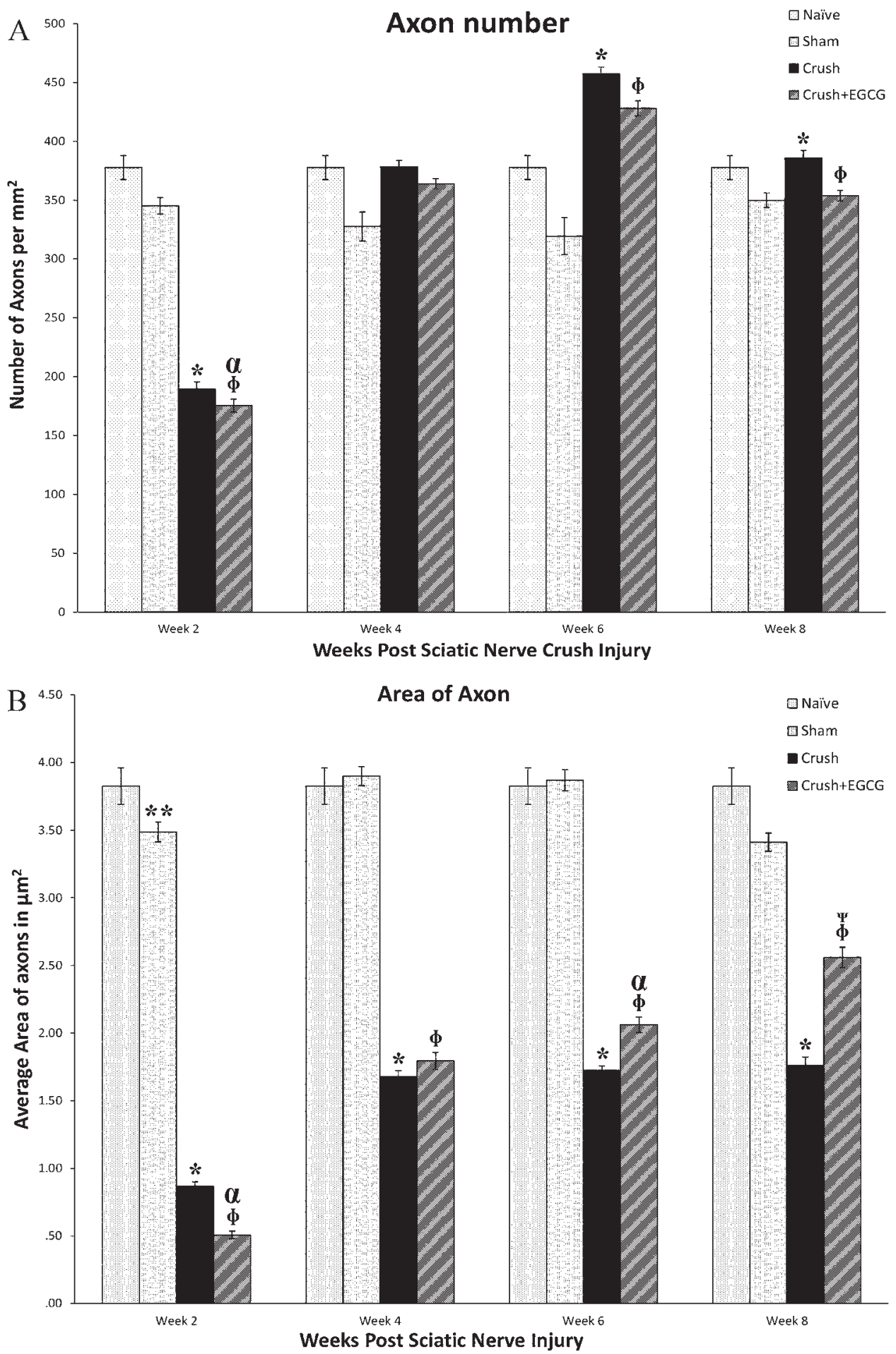

FIG. 5. A: The mean totals of myelinated axons counted from 10 different random samples from each animal used in the morphometric study. The mean total number of myelinated axons was calculated for each experimental group at Weeks $2,4,6$, and 8 following sciatic nerve injury using a $1-\mathrm{mm}^{2}$ square counting frame. * indicates $p<0.0001$, Crush vs Naïve, Sham, and Crush+EGCG; $\Phi$ indicates $p<0.0001$, Crush+EGCG vs Naïve, Sham, and Crush; $\alpha$ indicates $p<0.01$, Crush+EGCG vs Crush. B: The mean cross-sectional areas of myelinated axons were obtained from 10 different and randomly selected samples from each animal (3 animals/group). The averaged cross-sectional areas of myelinated axons measured in square micrometers $\left(\mu m^{2}\right)$ were calculated for each experimental group at Weeks 2, 4, 6, and 8 following sciatic nerve injuries. The Crush+EGCG animals showed a significant increase in the mean cross-sectional areas of the myelinated axons at Week 6 and Week 8 compared with the Crush group. * indicates $p<0.0001$, Crush vs Naïve and Sham; $\Phi$ indicates $p<0.0001$, Crush+EGCG vs Naïve, Sham, and Crush; $\alpha$ indicates $p<0.03$, Crush+EGCG vs Crush; $\Psi$ indicates $p<0.0001$, Crush+EGCG vs Crush; ** indicates $p<0.005$, Sham vs Naïve. Values are expressed as means and SDs (error bars). 

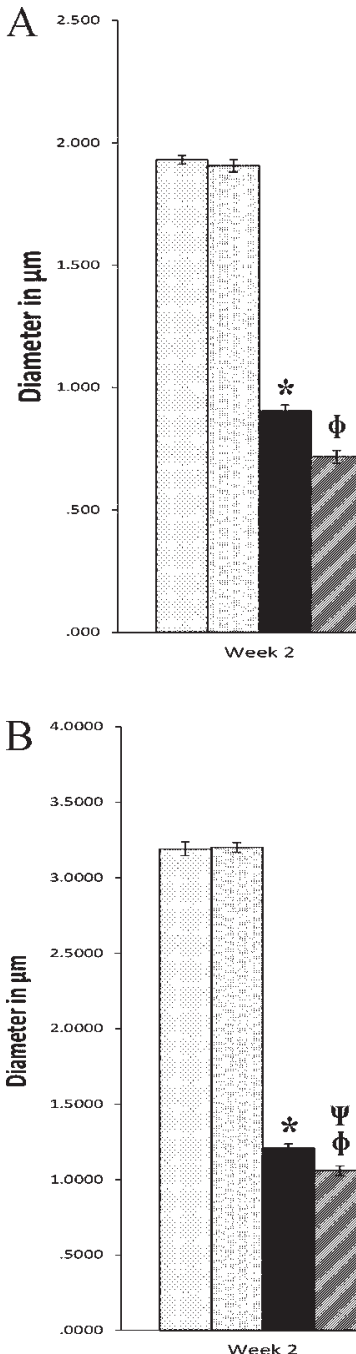

Nerve Fiber Diameter (D)

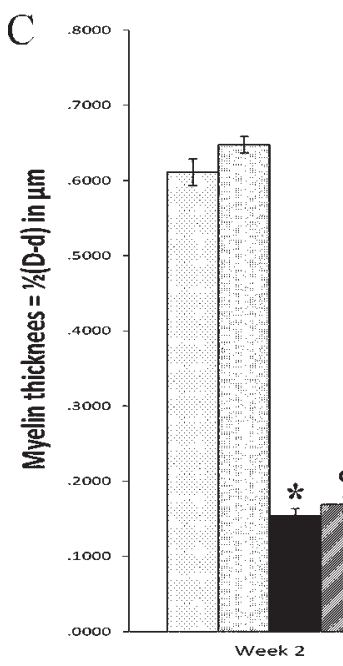

Axon diameter (d)

$a$

Myelin thickness
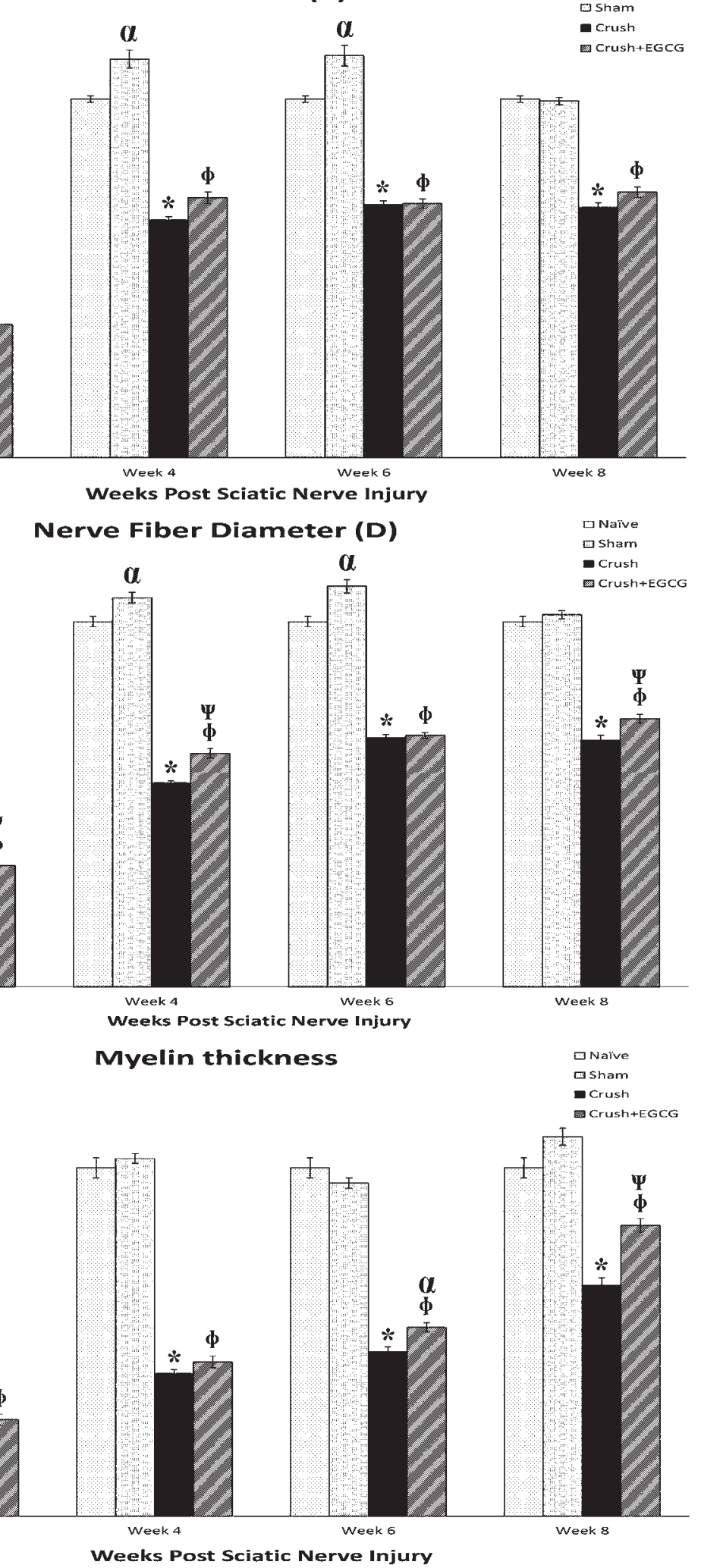

FIG. 6. Stereological estimation of the myelinated axon diameter $(\mathbf{A})$, myelinated nerve fiber diameter (B), and myelin thickness (C) obtained from 10 different random samples from each of 3 animals per group. The mean values of the axon diameters (d) and nerve fiber diameters (D) were calculated using an image analysis software package (Image-Pro Plus 6.0) for each experimental group at Weeks 2, 4, 6, and 8 following sciatic nerve injuries using a square counting frame. A: The Crush and Crush+EGCG groups displayed a significant decrease in axon diameter at all the time points assessed following nerve injury compared with Sham and Naïve animals. However, Crush+EGCG animals showed no significant change in comparison with Crush animals even at Week 8. ${ }^{*}$ indicates $p<$ 0.0001 , Crush vs Naïve and Sham; $\Phi$ indicates $p<0.0001$, Crush+EGCG vs Naïve and Sham; $\alpha$ indicates $p<$ 0.0001 , Sham vs Naïve. B: Likewise, the Crush and Crush+EGCG groups displayed a significant decrease in nerve fiber diameter at all the time points assessed following nerve injury compared with Sham and Naïve groups. The Crush+EGCG group showed a significant nerve fiber diameter increase by the end of Week 8 . * indicates $p<0.0001$, Crush vs Naïve and Sham; $\Phi$ indicates $p<0.0001$, Crush+EGCG vs Naïve and Sham; $\Psi$ indicates $p<0.008$, Crush+EGCG vs Crush; $\alpha$ indicates $p<0.001$, Sham vs Naïve. C: The calculated myelin thickness for the Crush and Crush+EGCG groups at different time points following sciatic nerve injury shows a significant decrease in myelin thickness throughout the experiment starting from Week 2 until Week 8. However, the myelin thickness in Crush+EGCG animals started to increase significantly at Week 6 and became more so at Week 8 compared with Crush group. ${ }^{*}$ indicates $p<0.0001$, Crush animals compared with Naïve and Sham groups; $\Phi$ indicates $p<0.0001$, Crush+EGCG animals compared with Naïve and Sham groups; $\Psi$ indicates $p<0.0001$, Crush+EGCG animals compared with Crush group; $\alpha$ indicates $p<0.05$, Crush+EGCG group compared with Crush group. Values are expressed as means and SDs (error bars).
Days 1,3 , and 14 postinjury compared with Crush group (Figs. 8D and 10E). RT-PCR analysis of MBP from spinal cord tissues revealed a significant $(\mathrm{p}<0.005)$ increase in
MBP gene expression at Days 7 and 14 following EGCG treatment compared with Crush animals (Fig. 8F).

The CV represents the ratio of the standard deviation 

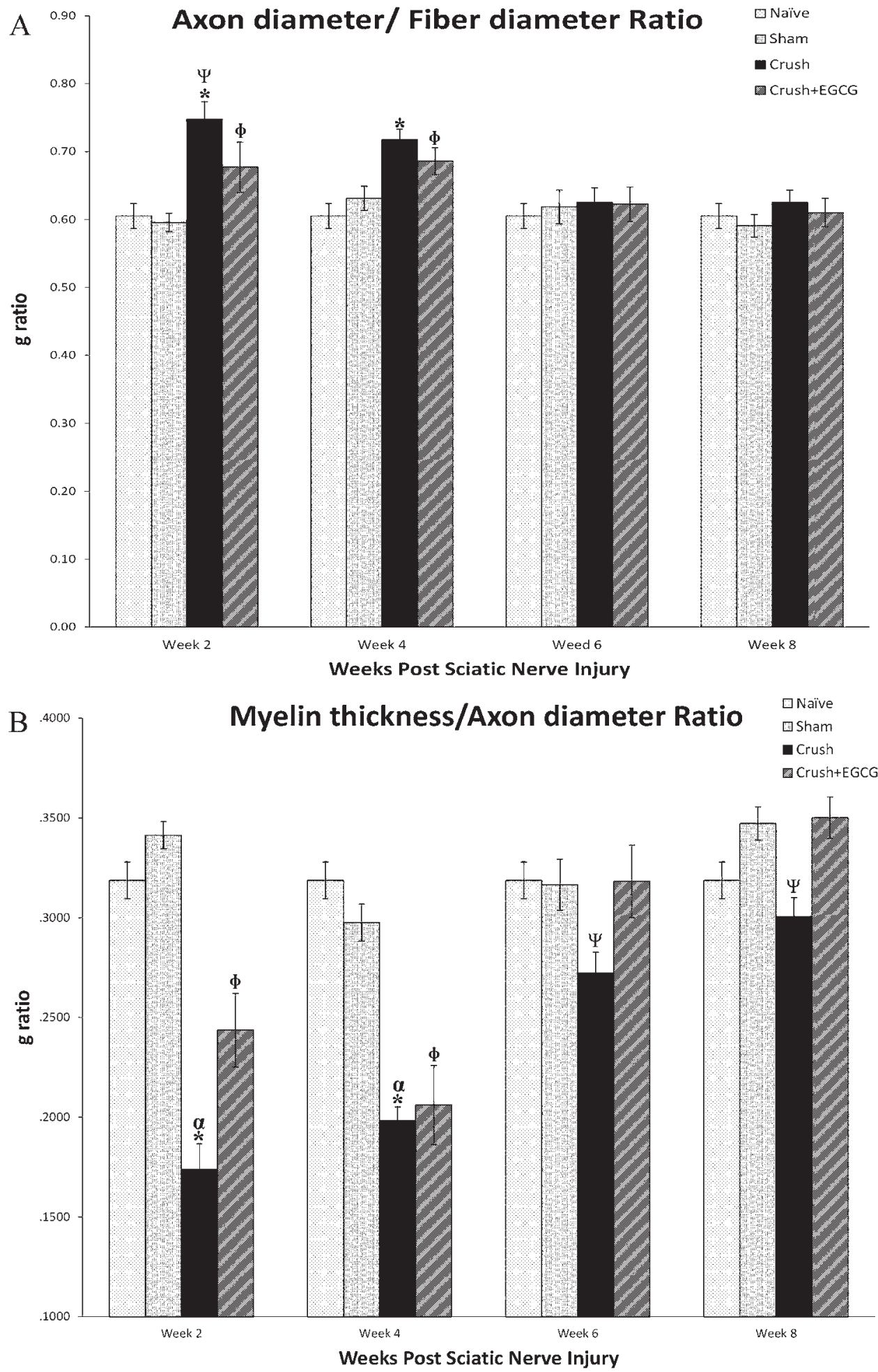

FIG. 7. Stereological analysis of the axon diameter to myelinated fiber diameter (g-ratio) $(\mathbf{A})$, myelinated nerve fiber diameter to axon diameter ratio (B), and myelin thickness to axon diameter ratio $(\mathbf{C})$ calculated from the data measurements obtained in 3 animals per group. The calculated data were averaged for each experimental group at Weeks 2, 4, 6, and 8 following sciatic nerve injuries. A: The Crush and Crush+EGCG groups showed a significant increase in the g-ratio at Week 2 and Week 4 postinjury. The Crush+EGCG animals displayed significantly lower g-ratio compared with Crush group only at Week 2 following injury. ${ }^{*}$ indicates $p<0.001$, Crush vs Naïve and Sham; $\Phi$ indicates $p<0.01$, Crush+EGCG vs Naïve and Sham; $\Psi$ indicates $p<0.05$, Crush vs Crush+EGCG. B: The Crush group showed a significant decrease in the myelin thickness to axon diameter ratio throughout the experiment period compared with all other groups, while the Crush+EGCG animals displayed a significantly lower fiber diameter to axon diameter ratio only at Week 2 and Week 8 compared with the Naïve and Sham groups. Also, the Crush+EGCG group significantly exhibited a higher fiber diameter to axon diameter ratio throughout the experimental period following injuries compared with the Crush group. * indicates $p<$ 0.0001 , Crush vs Naïve and Sham; $\Phi$ indicates $p<0.0001$, Crush+EGCG vs Naïve and Sham; $\alpha$ indicates $p$ $<0.001$, Crush vs Crush+EGCG group. $\Psi$ indicates $p<0.01$, Crush vs Crush+EGCG, Naïve, and Sham. Values are expressed as means and SDs (error bars). of the mean, and it is a useful statistic for comparing the degree of variation from one data series to another, even if the means are drastically different from each other. Analysis of the mean CVs for myelin thickness, axon diameter (d), and fiber diameter (D) for all the experimental groups at the different time points (Weeks 2, 4, 6, and 8) following sciatic nerve injury are given in Table 2 . At the end of the experiment, the CVs for myelin thickness, axon diameter (d), and fiber diameter (D) of Crush+EGCG group were lower in comparison with Crush animals. Simply put, the lower the ratio of standard deviation to mean return, the better the risk-return trade-off. 


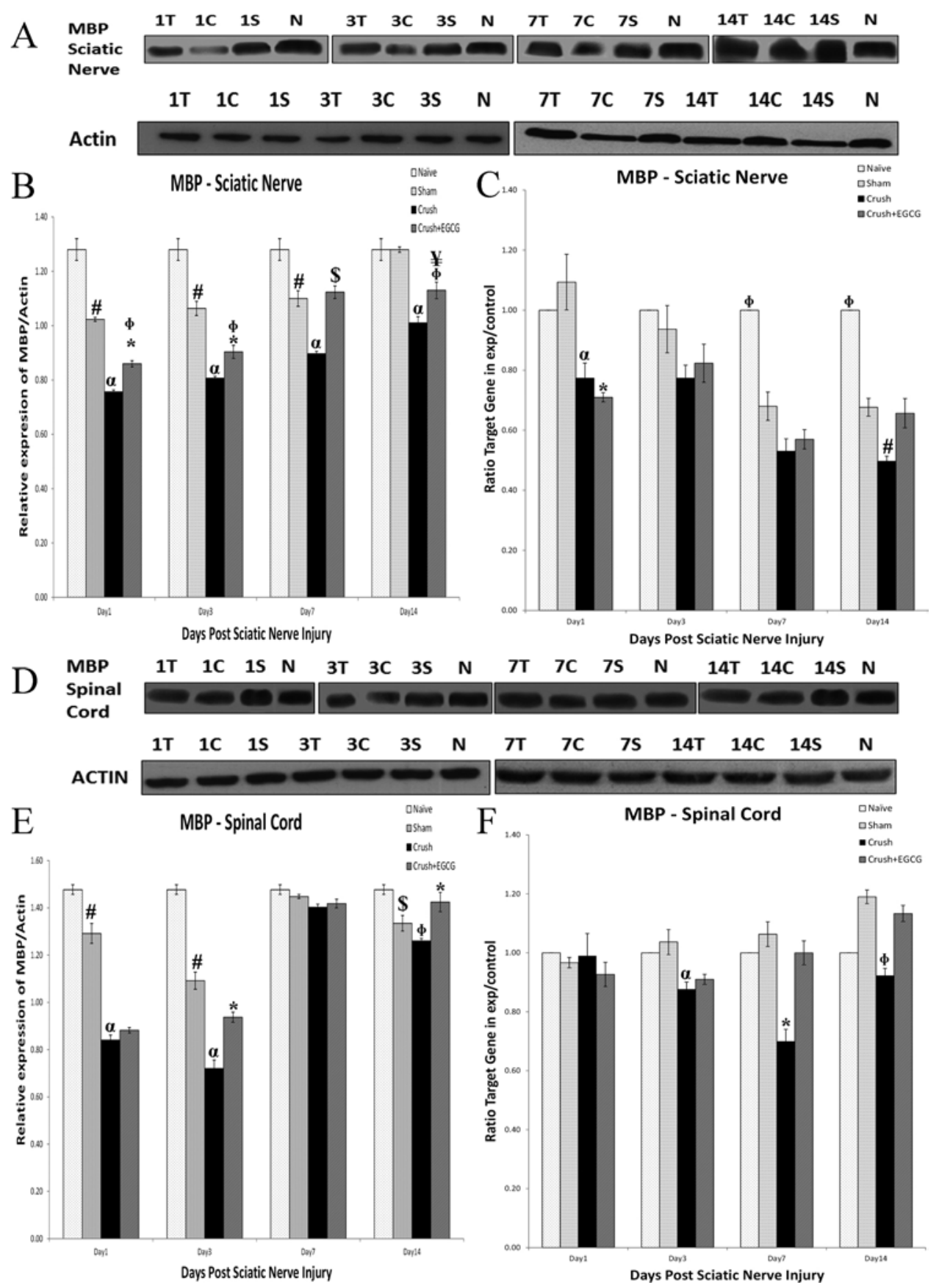

FIG. 8. Graphs of MBP Western blotting and RT-PCR gene expression analysis of the sciatic nerves and spinal cords at different time points following sciatic nerve crush injury. A and B: Western blot analysis of MBP in the sciatic nerves from all the experimental groups at different time points following nerve injury showing a significant increase in MBP concentration in the EGCG-treated animals from Day 1 until Day 14 postinjury compared with Crush group. \# indicates $p<0.01$, Sham vs Naïve; $\alpha$ indicates $p<0.0001$, Crush vs Sham and Naïve; * indicates $p<0.0001$, Crush+EGCG vs Naïve; $\Phi$ indicates $p<0.02$, Crush+EGCG vs Crush; $\$$ indicates $p<0.002$, Crush+EGCG vs Crush; $¥$ indicates $p<0.03$, Crush+EGCG vs Naïve and Sham. C: RT-PCR analysis of MBP from sciatic nerve tissues revealed a gradual increase in MBP gene expression at Day 3 and Day 7 following EGCG treatment. At Day 14, there was a significant increase in the MBP gene expression in the sciatic nerves of the EGCG-treated group compared with Crush animals. $\alpha$ indicates $p<0.03$, Crush vs Sham and Naïve; * indicates $p<0.01$, Crush+EGCG vs Naïve and Sham; $\Phi$ indicates $p<0.001$, Naïve vs Crush+EGCG, Crush, and Sham; \# indicates $p<0.01$, Crush vs Crush+EGCG and Sham. D and E: Western blot analysis of MBP in the spinal cords from all the experimental groups at different time points following nerve injury showing a significant increase in MBP concentration in the EGCG-treated animals at Day 1, Day 3, and Day 14 postinjury compared with the Crush group. $\alpha$ indicates $p<0.0001$, Crush vs Sham and Naïve; ${ }^{*}$ indicates $p<$ 0.005 , Crush+EGCG vs Crush; $\Phi$ indicates $p<0.004$, Crush vs Naïve; \# indicates $p<0.007$, Sham vs Naïve; $\$$ indicates $p<0.04$, Sham vs Naïve. F: RT-PCR analysis of MBP from spinal cord tissues revealed a significant increase in MBP gene expression (exp) at Day 7 and Day 14 following EGCG treatment compared with Crush animals. * indicates $p<0.005$, Crush vs Crush+EGCG, Sham, and Naïve; $\alpha$ indicates $p<0.02$, Crush vs Sham; $\Phi$ indicates $p<0.002$, Crush vs Crush+EGCG. Values are expressed as means and SDs (error bars). C = Crush; $\mathrm{N}=$ Naïve; $\mathrm{S}=$ Sham; $\mathrm{T}=$ Crush+EGCG; $1=$ Day 1; $3=$ Day 3; $7=$ Day 7; $14=$ Day 14. 
TABLE 2. Coefficients of variance for myelin thickness, axon diameter, and fiber diameter

\begin{tabular}{ccccc}
\hline Variable \& & \multicolumn{3}{c}{ Group } \\
\cline { 2 - 5 } Time Point & Naïve & Sham & Crush & Crush + EGCG \\
\hline Myelin thickness & & & \\
\hline Week 2 & 15.555 & 9.161 & 34.757 & 28.044 \\
\hline Week 4 & 15.555 & 7.468 & 14.761 & 20.543 \\
\hline Week 6 & 15.555 & 8.232 & 16.173 & 12.977 \\
\hline Week 8 & 15.555 & 10.038 & 17.093 & 12.586 \\
\hline Axon diameter & & & & \\
\hline Week 2 & 5.13 & 7.232 & 13.875 & 17.901 \\
\hline Week 4 & 5.13 & 12.628 & 7.763 & 12.177 \\
\hline Week 6 & 5.13 & 13.951 & 8.273 & 9.728 \\
\hline Week 8 & 4.591 & 9.572 & 10.508 & 5.13 \\
\hline Fiber diameter & & & & \\
\hline Week 2 & 7.878 & 5.652 & 12.046 & 14.698 \\
\hline Week 4 & 7.878 & 7.484 & 5.404 & 10.458 \\
\hline Week 6 & 7.878 & 9.234 & 6.584 & 6.354 \\
\hline Week 8 & 7.878 & 5.061 & 10.33 & 8.509 \\
\hline
\end{tabular}

\section{Oxidative Stress}

Attempts to determine lipid peroxidation products, the level of reduced glutathione, and activities of antioxidant enzymes in sciatic nerve specimen did not produce reliable data. The sample size measuring up to $10-15 \mathrm{~mm}$ in length and a few milligrams in weight was too small, and values were too low even when applying ultra-microassays.

\section{Isoprostanes}

Isoprostanes in urine were measured as an integrative marker of oxidative stress. Figure 9A shows isoprostane concentration in the urine of rats from different experimental groups. Samples were taken at Day 1, Day 7, Day 14, and Day 28 after the sciatic nerve injury. Statistically higher levels of isoprostanes were detected in the urine of sciatic nerve crushed rats at Day 7 after the injury, reaching a maximum at Day 14. Treatment with EGCG prevented the increase of isoprostanes.

\section{Total Antioxidant Capacity}

Changes in total antioxidant capacity (TAC) in the blood of different experimental groups at Day 1, Day 7, Day 14, and Day 28 after induction of the sciatic nerve injury are shown in Fig. 9B. Assessment of TAC, irrespective of its drawbacks, provides indirect information about the consumption of antioxidants as a result of oxidative stress. Figure 9B shows that the TAC level was significantly decreased in all groups except the control, irrespective of the treatment. TAC levels gradually recovered, and Sham and Crush groups reached control levels at Day 28 after the surgery. EGCG treatment accelerated recovery, and only in the EGCG-treated group was recovery complete at Day 14.

\section{Mechanisms of EGCG Action}

To study the beneficial effects of EGCG, the levels of expression of TNF- $\alpha$, IL-1 $\beta$, Nrf2, HO-1, and glutathione reductase were examined. We found that sciatic nerve crush induced the proinflammatory cytokine TNF- $\alpha$ in the spinal cord. Induction of TNF- $\alpha$ was first detected at 8 hours and reached a maximum at 16 hours after the injury. After that the level of the cytokine gradually decreased but remained significantly higher than in controls (Fig. 10A). Treatment with EGCG delayed the induction of TNF- $\alpha$, and at 8 hours postinjury the TNF- $\alpha$ level in the EGCG-treated animals was similar to that in controls. At 16 hours, however, no difference between the treated and the nontreated animals was observed, and at 24 hours the level of cytokine expression in the EGCG-treated group was higher than in the nontreated animals (Fig. 10A). The TNF- $\alpha$ level gradually decreased after reaching a maximum at 16 hours after the injury and was even much higher in controls at 72 hours.

In our experiments, sciatic nerve damage caused induction of Nrf2 mRNA in the spinal cord, reaching a maximum 8 hours after the injury and then returning to normal (Fig. 10B). A similar pattern was observed in the EGCGtreated animals, but the level of induction was much lower than in the Crush group (Fig. 10B).

Nrf2 regulates proteins/enzymes involved in protection against oxidative stress and inflammation. Among the proteins expressed by activated Nrf2 is HO-1. It catalyzes the oxidation of heme to biliverdin and also acts as a heatshock protein (HSP-32). ${ }^{55}$ Our data show that HO-1 was induced as a result of sciatic nerve damage. The induction was statistically significant 1 hour after the damage and gradually increased, reaching a maximum at 16 hours. Induction of HO-1 mRNA followed the same pattern in the EGCG-treated animals, but mRNA levels remained lower at all time points (Fig. 10C).

Levels of IL1- $\beta$ mRNA dropped in the 1st hour in all groups that underwent surgery and then progressively increased, reaching a maximum for the Crush group at 8 hours after the injury (Fig. 10D). A similar pattern was observed for the Crush+EGCG group, but EGCG treatment delayed the expression of IL1- $\beta$; the maximum was reached at 16 hours postinjury. Also, at all time points, the level of IL1- $\beta$ remained lower in the Crush+EGCG than in the Crush group.

Nerve crush caused mild induction of the enzyme glutathione reductase, presumably as a physiological response to increased glutathione oxidation (Fig. 10E). Dramatic induction of glutathione reductase was observed at 24 and 72 hours after injury in animals treated with EGCG (Fig. $10 \mathrm{E})$.

\section{Discussion}

The results of our study support the hypothesis that EGCG treatment is a potent stimulator of neuroregeneration after crush injury of the sciatic nerve. EGCG treatment substantively improved several sensory and motor parameters as well as mended the morphological alterations after nerve crush injury. The results of the current work are consistent with our previous studies and other investigations that examined the neuroprotective properties of EGCG on peripheral neuropathies, ${ }^{36,44,51,63}$ Parkin- 



FIG. 9. Concentration of isoprostanes in urine and blood TAC. Samples from Naïve, Sham, Crush, and Crush+EGCG experimental groups were collected at Days 1, 7, 14, and 28 after the induction of the sciatic nerve injury followed by treatment as indicated in the methodology protocol. A: Urine concentrations of isoprostanes. Data are presented as mean \pm SEM. * indicates $p<0.05$, Crush vs Naïve and Sham; $\Phi$ indicates $p<0.0001$, Crush vs Naïve, Sham, and Crush+EGCG. B: TAC in the blood. * indicates $p<$ 0.003 , Naïve vs Sham, Crush, and Crush+EGCG; $\Phi$ indicates $p<0.05$, Naïve and Crush+EGCG vs Sham and Crush.

son's disease, Alzheimer's disease, and traumatic brain injury. ${ }^{26,36,54}$

Our results provide further evidence for injury-related pain-relieving and motor-repair effects of EGCG in a posttraumatic sciatic nerve injury rat model. The functional motor recovery of the affected limb showed a progressive improvement in foot position, toe spread, EPT, and hopping tests starting from Week 1 and continuing up until the end of Week 8 after nerve injury; these results are in complete agreement with our recent studies using rat models of sciatic crush nerve injury and spinal cord injury. ${ }^{48,50,51}$

EGCG neurobehavioral recovery was well supported by the current and previous morphological improvements in the histological architecture of the crush-injured nerves and the increased survival of neurons in both the dorsal and ventral horns of spinal cords of EGCG-treated rats on 

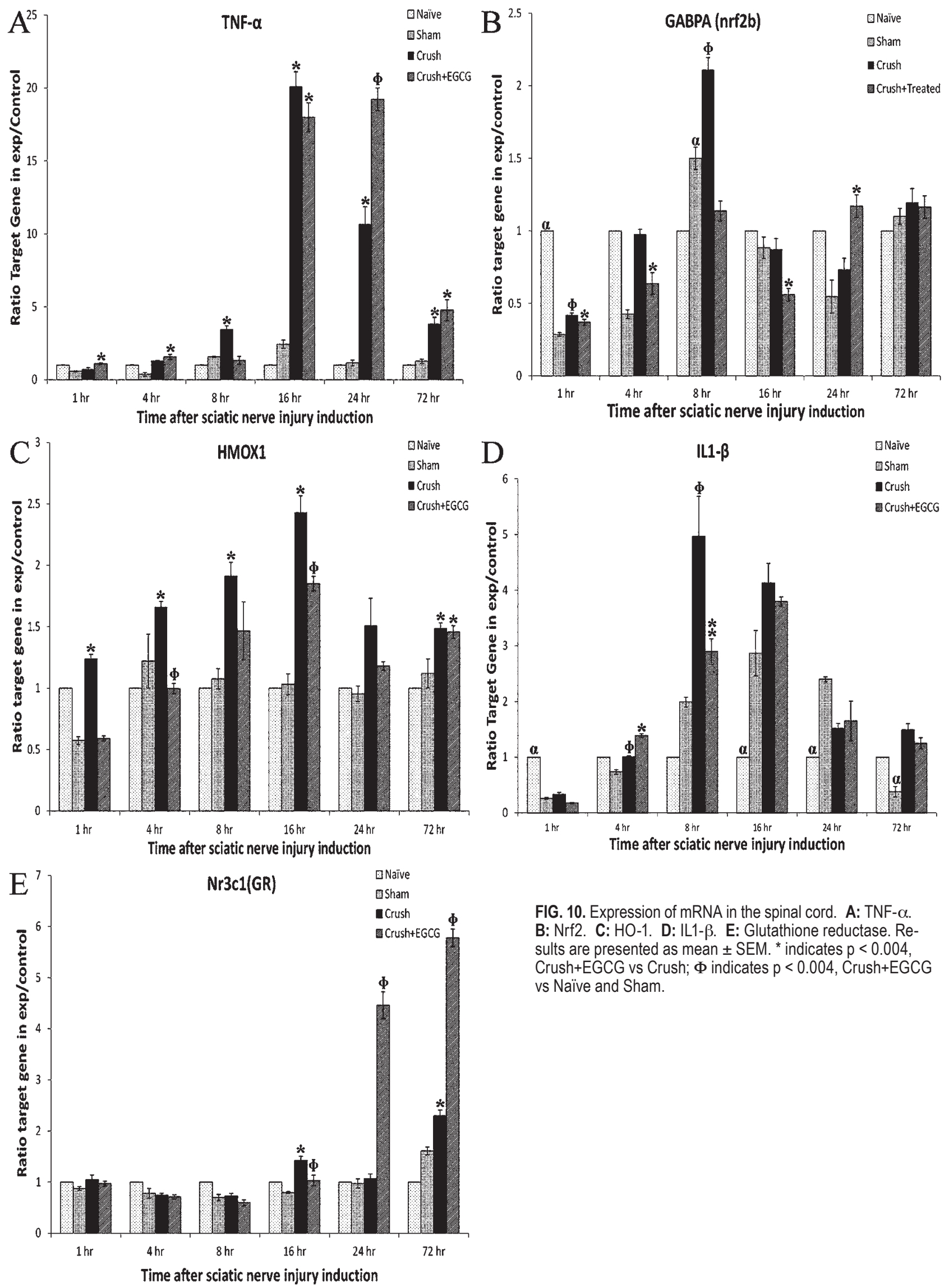

FIG. 10. Expression of mRNA in the spinal cord. A: TNF- $\alpha$. B: Nrf2. C: HO-1. D: IL1- $\beta$. E: Glutathione reductase. Results are presented as mean \pm SEM. * indicates $p<0.004$, Crush+EGCG vs Crush; $\Phi$ indicates $p<0.004$, Crush+EGCG vs Naïve and Sham. 
histological examination. ${ }^{50,51}$ Recently, it has been shown that intraperitoneal injection of EGCG $(60 \mathrm{mg} / \mathrm{kg}) \mathrm{im}-$ proved the sensory allodynia and thermal thresholds in animals with oxaliplatin-induced peripheral neuropathy. ${ }^{33}$ Similarly, chronic oral administration of epigallocatechingallate alleviates streptozotocin-induced diabetic neuropathic hyperalgesia in rats with a concomitant decrease of 3,4-methylenedioxyamphetamine (MDA) and nitrite content and an increase of erythrocyte superoxide dismutase activity as blood markers of oxidative stress..$^{4,55}$

Our neurobehavioral pain assessment results are consistent with many recent studies displaying EGCG modulation of pain parameters in different models of neuropathy. A recent study showed that an aqueous solution of EGCG in the drinking water ameliorated mechanical hypersensitivity in a model of streptozotocin-induced diabetic neuropathic pain ${ }^{47}$ and in the posttraumatic osteoarthritis mouse model. ${ }^{35}$ Moreover, mice treated with EGCG showed reduced osteoarthritis-associated pain, as indicated by higher locomotor behavior (i.e., distance traveled) with a significant concurrent reduction in the expression levels of the chemokine receptor, and proinflammatory cytokines IL-1 $\beta$ and TNF-1 $\alpha$ in the dorsal root ganglia similar to sham-operated animals. ${ }^{35}$ Likewise, intrathecal EGCG injection modulated the nociceptive progression and the reactive oxygen species, ${ }^{2}$ the nNOS protein expression and the pronociceptive effects of $\mathrm{NO}^{11}$ in rats with spinal nerve ligation neuropathic pain model. Further, intrathecal injection of EGCG in an animal model of sciatic nerve chronic constriction injury was associated with marked improvement in pain behaviors and decreased expressions of Toll-like receptor TLR4, High Mobility Group 1 protein (HMGB1), NF-кB, TNF- $\alpha$, and IL-1 $\beta$ along with a significant increase in IL-10 content. ${ }^{31}$

Histological examination of semi-thin transverse sections of sciatic nerves from EGCG-treated animals revealed a remarkable axonal and myelin regeneration. Compared with sciatic nerve injury group, these sections contained a greater number of large newly regenerated nerve fibers that were surrounded by much more myelin. Sciatic nerves of the EGCG-treated group reached almost normal morphology by the end of Week 8 postsurgery in contrast to Crush sciatic nerve rats. These results are consistent with the early light and ultrastructural evaluation of EGCG-treated sciatic nerves that showed normal unmyelinated and myelinated axons with regular myelin sheath thickness and normalized appearance of SchmidtLantermann clefts. ${ }^{50}$ Furthermore, these results are in line with studies showing EGCG being a neuroprotective and neuroregenerative agent in an experimental model of optic nerve crush injury. ${ }^{46,74}$ Quantitative estimation and stereological analysis of myelinated nerve fiber number, fiber size parameters, and myelin and axonal thickness are essential tools for nerve regeneration investigation. The Crush and Crush+EGCG groups showed a significant decrease in the mean cross-sectional areas of myelinated axons compared with control groups throughout the experiment period. However, the EGCG-treated group showed a significant increase in nerve fiber diameter size, mean areas of the myelinated axons, and myelin thickness starting at Week 6 compared with the Crush group. Biochemically, this was also confirmed by the increase of MBP concentration and gene expression in both the injured sciatic nerve and spinal cord following EGCG treatment. Recently, we have shown a significant decrease in the number of neurons at the L4-L5 spinal cord levels (corresponding to the sciatic nerve origin) in the Crush group, compared with the Naïve and Sham groups. ${ }^{51}$ Further, EGCG treatment increased the number of motor neurons by reducing the expression of the glial fibrillary acidic protein and increased the expression of growth-associated protein-43, a marker of regenerating axons. EGCG treatment also reduced the ratio of B-cell lymphoma 2 (Bcl-2)-associated $\mathrm{X}$ protein/Bcl-2 and increased the expression of survivin gene indicating a possible role of EGCG in modulating the apoptotic pathway to protect the neurons. ${ }^{51}$ Therefore, it seems reasonable that MBP is downregulated centrally in the Crush animals, due to loss of neurons and their axons while the EGCG treatment upregulated it to restore the damaged myelin or to protect the myelin from disintegrations following a neuronal loss in the spinal cord after nerve injury.

The higher number of small fibers observed, especially in EGCG-treated and untreated regenerated crushed nerve, is in agreement with the sprouting phenomenon, which is considered a hallmark of regeneration during and after axonal degeneration. ${ }^{41,55}$ Keilhoff et al. ${ }^{30}$ reported an increase in the number of immature and newly generated myelin fibers at 8 weeks after nerve injury signifying persistent uncontrolled (futile) sprouting. Injured axons produced dozens of collateral nerve sprouts at the injury site during the initial stage of peripheral nerve regeneration. However, few enter the distal nerve stump due to the limitation of the number of pathfinding structures (bands of Büngner). Many small nerve fibers with a healthy histomorphology, though still ensheathed with a thin myelin layer, may be designated as regenerated nerve fibers that have already begun the myelination process. The healthy unmyelinated nerve fiber bundles found in the crushed nerve can be categorized as either regenerated unmyelinated fibers or regenerated nerve fibers that did not reach the myelination processes. $^{70}$

Analysis of the g-ratios in our study revealed that in the Crush+EGCG group the treatment significantly lowered the axon diameter to myelinated fiber diameter ratio and myelin thickness to axon diameter ratio compared with Crush group values at Week 2 and Week 4 postinjury before normalization with control values. Our morphometric data analysis supports our hypothesis that EGCG has a profound positive effect on the myelinated axons. As the g-ratio is determined by the ratio of the axon to fiber diameter, a higher g-ratio can be expected in a demyelinating neuropathy, as remyelination leads to thinner myelin sheaths while the axon diameter remains the same. This lower axon diameter to myelinated fiber diameter ratio in our study can be due to either the presence of regenerated fibers with a thin myelin sheath in injured nerves, resulting in higher g-ratios or the loss of small myelinated nerve fibers in injured nerves, as small myelinated nerve fibers have higher g-ratios than large myelinated nerve fibers. ${ }^{15,27}$ Our g-ratio analysis is consistent with those of many studies, and it provides verification of the efficacy of the 
EGCG treatment in improving and speeding the regeneration process in the crush-injured sciatic nerves.

Tissue damage and disruption of homeostasis are among the known inducers of oxidative stress. In our study, sciatic nerve crush injury produced generalized oxidative stress, manifested as an increase in isoprostane levels in urine and a decrease of TAC of the blood. A rapid increase of isoprostane levels in the brain tissue as the result of traumatic brain injury has been previously reported ${ }^{66}$ and was accompanied by a concomitant decrease of brain antioxidant reserves and increased production of proinflammatory mediators. Induction of a proinflammatory cytokine, TNF- $\alpha$, was detected in our experiments and was attributable to traumatic damage to the sciatic nerve. A statistically significant increase in urinary isoprostanes was detected at Day 7 postinjury and reached a maximum at Day 14. Such dynamics followed the decrease of myelinated axons. Judging by the urinary excretion of isoprostanes, treatment with EGCG prevented crush-induced generalized oxidative stress and accelerated the restoration of the antioxidant capacity, which coincided with morphological improvement of the nerve. Based on the data for induction of TNF- $\alpha$, the beneficial effect of EGCG does not seem to be related to the decrease in proinflammatory cytokines. It is known, however, that polyphenols, including EGCG, can suppress oxidative stress by affecting transcription factors regulating the expression of antioxidant enzymes. Among such transcription factors is nuclear factor erythroid 2-related factor 2 (Nrf2). ${ }^{42}$ The redox-sensitive Nrf2-antioxidant response element (ARE) pathway is considered the main sensor and major suppressor of oxidative stress through upregulation of the expression of a large number of genes coding for antioxidant enzymes. ${ }^{16}$ We found that Nrf2 in the spinal cord was induced very early after sciatic nerve damage and that EGCG treatment suppressed the induction. Nrf2 is supposedly activated by oxidation of cysteine residues or by phosphorylation. Recent findings indicate that protective effects of EGCG can be mediated via activation of $\mathrm{Nrf} 2$, and as a consequence, induction of antioxidant enzymes, including superoxide dismutase (SOD), catalase, glutathione- $S$-transferase, glutathione peroxidase, etc..$^{18}$ Our results suggest that the beneficial effect of EGCG is not mediated via Nrf2 induction in the spinal cord. On the other hand, EGCG treatment induced glutathione reductase, which plays a key role in maintaining glutathione in its functional reduced state.

Another enzyme that is controlled by redox-sensitive transcription factors is the inducible form of heme oxygenase, HO-1. Its induction is considered an adaptive response to oxidative stress and inflammation. $.^{45} \mathrm{HO}-1$ was induced very early in the spinal cords of animals with crushed sciatic nerves, and EGCG treatment suppressed its induction. EGCG treatment of sciatic nerve-injured rats was also recently shown to enhance the survival of spinal cord neurons by modulating the expression of neuronal growth genes along with apoptotic and antiapoptotic genes, thus conserving the spinal cord neurons..$^{49,51}$

Recent in vitro studies provide evidence for the antioxidative and neuroprotective activity of EGCG in aging-associated oxidative damage in rat brain by decreasing the production of hydroxynonenal in the aged brain, upregulating the antioxidant system, and augmenting the activities of Kreb's cycle enzymes and electron transport chain complexes in aged brain mitochondria. ${ }^{60}$ Likewise, oral administration of EGCG was associated with significant improvement in cognitive deficits in mice and elevated total superoxide dismutase and glutathione peroxidase activities, decreased 3,4-methylenedioxyamphetamine contents in the hippocampus, and reduced the cell apoptosis index in the mouse hippocampus. Further, oral administration of EGCG in mice has been shown to enhance cell division and increase the number of neuroblasts in the dentate gyrus ${ }^{75}$ and improve cognitive dysfunction induced by psychological stress through suppressing lipid peroxidation and production of reactive oxidative species and increasing total antioxidative capacity. ${ }^{9}$ This green tea modulation may also result from changes in 5-hydroxytryptamine, cytokines, and catecholamines, and expressions of metallothioneins. ${ }^{10}$

\section{Conclusions}

In summary, EGCG-treated animals displayed a remarkable decrease in nociception parameters compared with Crush group starting at Week 2 and maintained until the end of the experiment at Week 10. The results of our sensory neurobehavioral tests-allodynia time latency, paw pressure latency (mechanical hyperalgesia), WRL using a hot plate analgesia meter, and central nociception measured as tail flick withdrawal latency-are consistent with our previous studies using the same crush injury of the sciatic nerve rat model as well as with other investigations using EGCG treatment in different models of neuropathy. Also, our quantitative stereological evaluation results reveal significant histomorphological evidence of neuronal regeneration in the EGCG-treated injured animals compared with control groups throughout the duration of the experiment. Only upregulation of glutathione reductase supports the idea that EGCG is acting indirectly via induction of enzymes or transcription factors. Although our study clearly supports the beneficial role of EGCG as a neuroprotective agent and a facilitator of neuronal regeneration, further investigations are needed to establish whether antioxidative mechanisms are involved in the regenerative process after nerve crush injury.

\section{Acknowledgments}

We would like to thank Mr. Jijin Kumar, Mrs. Preethi George, Mrs. Sinu Joe, Mrs. Soumya Jose, and Mrs. Shabeeba Pattillath for their excellent technical assistance. Also, we acknowledge the support of the Animal Resource Center, Health Science Center, Kuwait University. This work was funded by Kuwait University Research Administration (grant No. MA01/11) and Research Sector OMICSRU grant SRUL02/13.

\section{References}

1. Akhtar N, Haqqi TM: Epigallocatechin-3-gallate suppresses the global interleukin-lbeta-induced inflammatory response in human chondrocytes. Arthritis Res Ther 13:R93, 2011

2. An SS, Kim YO, Park CH, Lin H, Yoon MH: Antiallodynic effect of intrathecal epigallocatechin-3-gallate due to suppression of reactive oxygen species. Korean J Anesthesiol 67:123-128, 2014 
3. Aslan H, Altunkaynak BZ, Altunkaynak ME, Vuraler O, Kaplan S, Unal B: Effect of a high fat diet on quantitative features of adipocytes in the omentum: an experimental, stereological and ultrastructural study. Obes Surg 16:15261534,2006

4. Baluchnejadmojarad T, Roghani M: Chronic oral epigallocatechin-gallate alleviates streptozotocin-induced diabetic neuropathic hyperalgesia in rat: involvement of oxidative stress. Iran J Pharm Res 11:1243-1253, 2012

5. Bélanger E, Henry FP, Vallée R, Randolph MA, Kochevar IE, Winograd JM, et al: In vivo evaluation of demyelination and remyelination in a nerve crush injury model. Biomed Opt Express 2:2698-2708, 2011

6. Cai J, Jing D, Shi M, Liu Y, Lin T, Xie Z, et al: Epigallocatechin gallate (EGCG) attenuates infrasoundinduced neuronal impairment by inhibiting microgliamediated inflammation. J Nutr Biochem 25:716-725, 2014

7. Canan S, Aktaş A, Ulkay MB, Colakoglu S, Ragbetli MC, Ayyildiz M, et al: Prenatal exposure to a non-steroidal antiinflammatory drug or saline solution impairs sciatic nerve morphology: a stereological and histological study. Int J Dev Neurosci 26:733-738, 2008

8. Canan S, Bozkurt HH, Acar M, Vlamings R, Aktas A, Sahin B, et al: An efficient stereological sampling approach for quantitative assessment of nerve regeneration. Neuropathol Appl Neurobiol 34:638-649, 2008

9. Chen WQ, Zhao XL, Hou Y, Li ST, Hong Y, Wang DL, et al: Protective effects of green tea polyphenols on cognitive impairments induced by psychological stress in rats. Behav Brain Res 202:71-76, 2009

10. Chen WQ, Zhao XL, Wang DL, Li ST, Hou Y, Hong Y, et al: Effects of epigallocatechin-3-gallate on behavioral impairments induced by psychological stress in rats. Exp Biol Med (Maywood) 235:577-583, 2010

11. Choi JI, Kim WM, Lee HG, Kim YO, Yoon MH: Role of neuronal nitric oxide synthase in the antiallodynic effects of intrathecal EGCG in a neuropathic pain rat model. Neurosci Lett 510:53-57, 2012

12. Choi YB, Kim YI, Lee KS, Kim BS, Kim DJ: Protective effect of epigallocatechin gallate on brain damage after transient middle cerebral artery occlusion in rats. Brain Res 1019:47-54, 2004

13. De Vry J, Kuhl E, Franken-Kunkel P, Eckel G: Pharmacological characterization of the chronic constriction injury model of neuropathic pain. Eur J Pharmacol 491:137-148, 2004

14. Francisco JS, Moraes HP, Dias EP: Evaluation of the ImagePro Plus 4.5 software for automatic counting of labeled nuclei by PCNA immunohistochemistry. Braz Oral Res 18:100-104, 2004

15. Friede RL, Beuche W: Combined scatter diagrams of sheath thickness and fibre calibre in human sural nerves: changes with age and neuropathy. J Neurol Neurosurg Psychiatry 48:749-756, 1985

16. Gan L, Johnson JA: Oxidative damage and the Nrf2-ARE pathway in neurodegenerative diseases. Biochim Biophys Acta 1842:1208-1218, 2014

17. Geuna S: The revolution of counting "tops": two decades of the disector principle in morphological research. Microsc Res Tech 66:270-274, 2005

18. Geuna S, Tos P, Guglielmone R, Battiston B, GiacobiniRobecchi MG: Methodological issues in size estimation of myelinated nerve fibers in peripheral nerves. Anat Embryol (Berl) 204:1-10, 2001

19. Gundersen RY, Vaagenes P, Breivik T, Fonnum F, Opstad PK: Glycine - an important neurotransmitter and cytoprotective agent. Acta Anaesthesiol Scand 49:1108-1116, 2005

20. Hadlock TA, Koka R, Vacanti JP, Cheney ML: A comparison of assessments of functional recovery in the rat. J Peripher Nerv Syst 4:258-264, 1999

21. Haque AM, Hashimoto M, Katakura M, Hara Y, Shido O: Green tea catechins prevent cognitive deficits caused by $\mathrm{A} \beta_{1-40}$ in rats. J Nutr Biochem 19:619-626, 2008

22. He M, Zhao L, Wei MJ, Yao WF, Zhao HS, Chen FJ: Neuroprotective effects of (-)-epigallocatechin-3-gallate on aging mice induced by D-galactose. Biol Pharm Bull 32:5560,2009

23. Higdon JV, Frei B: Tea catechins and polyphenols: health effects, metabolism, and antioxidant functions. Crit Rev Food Sci Nutr 43:89-143, 2003

24. Hirata K, He JW, Kuraoka A, Omata Y, Hirata M, Islam ATMS, et al: Heme oxygenasel (HSP-32) is induced in myelin-phagocytosing Schwann cells of injured sciatic nerves in the rat. Eur J Neurosci 12:4147-4152, 2000

25. Huang GS, Tseng CY, Lee CH, Su SL, Lee HS: Effects of (-)-epigallocatechin-3-gallate on cyclooxygenase 2, $\mathrm{PGE}_{2}$, and IL-8 expression induced by IL-1beta in human synovial fibroblasts. Rheumatol Int 30:1197-1203, 2010

26. Itoh $T$, Imano $M$, Nishida $S$, Tsubaki $M$, Hashimoto $S$, Ito $A$, et al: (-)-Epigallocatechin-3-gallate protects against neuronal cell death and improves cerebral function after traumatic brain injury in rats. Neuromolecular Med 13:300-309, 2011

27. Jacobs JM, Love S: Qualitative and quantitative morphology of human sural nerve at different ages. Brain 108:897-924, 1985

28. Jiang J, Mo ZC, Yin K, Zhao GJ, Lv YC, Ouyang XP, et al: Epigallocatechin-3-gallate prevents TNF- $\alpha$-induced NF- $\kappa B$ activation thereby upregulating ABCA1 via the Nrf2/Keap1 pathway in macrophage foam cells. Int J Mol Med 29:946956, 2012

29. Kaplan S, Gökyar A, Unal B, Tunç AT, Bahadir A, Aslan $\mathrm{H}$ : A simple technique for localizing consecutive fields for disector pairs in light microscopy: application to neuron counting in rabbit spinal cord following spinal cord injury. $\mathbf{J}$ Neurosci Methods 145:277-284, 2005

30. Keilhoff G, Langnaese K, Wolf G, Fansa H: Inhibiting effect of minocycline on the regeneration of peripheral nerves. Dev Neurobiol 67:1382-1395, 2007

31. Kuang X, Huang Y, Gu HF, Zu XY, Zou WY, Song ZB, et al: Effects of intrathecal epigallocatechin gallate, an inhibitor of Toll-like receptor 4, on chronic neuropathic pain in rats. Eur J Pharmacol 676:51-56, 2012

32. Kubo K, Nishikawa K, Ishizeki J, Hardy-Yamada M, Yanagawa Y, Saito S: Thermal hyperalgesia via supraspinal mechanisms in mice lacking glutamate decarboxylase 65. J Pharmacol Exp Ther 331:162-169, 2009

33. Lee JS, Kim YT, Jeon EK, Won HS, Cho YS, Ko YH: Effect of green tea extracts on oxaliplatin-induced peripheral neuropathy in rats. BMC Complement Altern Med 12:124, 2012

34. Lee SY, Kim CY, Lee JJ, Jung JG, Lee SR: Effects of delayed administration of (-)-epigallocatechin gallate, a green tea polyphenol on the changes in polyamine levels and neuronal damage after transient forebrain ischemia in gerbils. Brain Res Bull 61:399-406, 2003

35. Leong DJ, Choudhury M, Hanstein R, Hirsh DM, Kim SJ, Majeska RJ, et al: Green tea polyphenol treatment is chondroprotective, anti-inflammatory and palliative in a mouse post-traumatic osteoarthritis model. Arthritis Res Ther 16:508, 2014

36. Liu M, Chen F, Sha L, Wang S, Tao L, Yao L, et al: (-)-Epigallocatechin-3-gallate ameliorates learning and memory deficits by adjusting the balance of TrkA/p75NTR signaling in APP/PS1 transgenic mice. Mol Neurobiol 49:1350-1363, 2014

37. Luís AL, Amado S, Geuna S, Rodrigues JM, Simões MJ, Santos JD, et al: Long-term functional and morphological 
assessment of a standardized rat sciatic nerve crush injury with a non-serrated clamp. J Neurosci Methods 163:92-104, 2007

38. Mandel S, Weinreb O, Amit T, Youdim MB: Cell signaling pathways in the neuroprotective actions of the green tea polyphenol (-)-epigallocatechin-3-gallate: implications for neurodegenerative diseases. J Neurochem 88:1555-1569, 2004

39. Mendonça AC, Barbieri CH, Mazzer N: Directly applied low intensity direct electric current enhances peripheral nerve regeneration in rats. J Neurosci Methods 129:183-190, 2003

40. Moore AM, Borschel GH, Santosa KA, Flagg ER, Tong AY, Kasukurthi R, et al: A transgenic rat expressing green fluorescent protein (GFP) in peripheral nerves provides a new hindlimb model for the study of nerve injury and regeneration. J Neurosci Methods 204:19-27, 2012

41. Mozaffar T, Strandberg E, Abe K, Hilgenberg LG, Smith MA, Gupta R: Neuromuscular junction integrity after chronic nerve compression injury. J Orthop Res 27:114-119, 2009

42. Na HK, Kim EH, Jung JH, Lee HH, Hyun JW, Surh YJ: (-)-Epigallocatechin gallate induces Nrf2-mediated antioxidant enzyme expression via activation of PI3K and ERK in human mammary epithelial cells. Arch Biochem Biophys 476:171-177, 2008

43. Na HK, Surh YJ: Modulation of Nrf2-mediated antioxidant and detoxifying enzyme induction by the green tea polyphenol EGCG. Food Chem Toxicol 46:1271-1278, 2008

44. Ownby SL, Fortuno LV, Au AY, Grzanna MW, RashmirRaven AM, Frondoza CG: Expression of pro-inflammatory mediators is inhibited by an avocado/soybean unsaponifiables and epigallocatechin gallate combination. J Inflamm (Lond) 11:8, 2014

45. Paine A, Eiz-Vesper B, Blasczyk R, Immenschuh S: Signaling to heme oxygenase-1 and its anti-inflammatory therapeutic potential. Biochem Pharmacol 80:1895-1903, 2010

46. Peng PH, Chiou LF, Chao HM, Lin S, Chen CF, Liu JH, et al: Effects of epigallocatechin-3-gallate on rat retinal ganglion cells after optic nerve axotomy. Exp Eye Res 90:528-534, 2010

47. Raposo D, Morgado C, Pereira-Terra P, Tavares I: Nociceptive spinal cord neurons of laminae I-III exhibit oxidative stress damage during diabetic neuropathy which is prevented by early antioxidant treatment with epigallocatechin-gallate (EGCG). Brain Res Bull 110:68-75, 2015

48. Renno WM, Al-Khaledi G, Mousa A, Karam SM, Abul H, Asfar S: (-)-Epigallocatechin-3-gallate (EGCG) modulates neurological function when intravenously infused in acute and, chronically injured spinal cord of adult rats. Neuropharmacology 77:100-119, 2014

49. Renno WM, Al-Maghrebi M, Al-Banaw A:

(-)-Epigallocatechin-3-gallate (EGCG) attenuates functional deficits and morphological alterations by diminishing apoptotic gene overexpression in skeletal muscles after sciatic nerve crush injury. Naunyn Schmiedebergs Arch Pharmacol 385:807-822, 2012

50. Renno WM, Al-Maghrebi M, Alshammari A, George P: (-)-Epigallocatechin-3-gallate (EGCG) attenuates peripheral nerve degeneration in rat sciatic nerve crush injury. Neurochem Int 62:221-231, 2013

51. Renno WM, Al-Maghrebi M, Rao MS, Khraishah H: (-)-Epigallocatechin-3-gallate modulates spinal cord neuronal degeneration by enhancing growth-associated protein 43, B-cell lymphoma 2 , and decreasing B-cell lymphoma 2-associated $\mathrm{x}$ protein expression after sciatic nerve crush injury. J Neurotrauma 32:170-184, 2015

52. Renno WM, Khan KM, Benov L: Is there a role for neurotrophic factors and their receptors in augmenting the neuroprotective effect of (-)-epigallocatechin-3-gallate treatment of sciatic nerve crush injury? Neuropharmacology 102:1-20, 2016

53. Renno WM, Saleh F, Klepacek I, Al-Khaledi G, Ismael H, Asfar S: Green tea pain modulating effect in sciatic nerve chronic constriction injury rat model. Nutr Neurosci 9:4147, 2006

54. Rezai-Zadeh K, Shytle D, Sun N, Mori T, Hou H, Jeanniton D, et al: Green tea epigallocatechin-3-gallate (EGCG) modulates amyloid precursor protein cleavage and reduces cerebral amyloidosis in Alzheimer transgenic mice. $\mathbf{J}$ Neurosci 25:8807-8814, 2005

55. Ronchi G, Jager SB, Vaegter CB, Raimondo S, GiacobiniRobecchi MG, Geuna S: Discrepancies in quantitative assessment of normal and regenerated peripheral nerve fibers between light and electron microscopy. J Peripher Nerv Syst 19:224-233, 2014

56. Savastano LE, Laurito SR, Fitt MR, Rasmussen JA, Gonzalez Polo V, Patterson SI: Sciatic nerve injury: a simple and subtle model for investigating many aspects of nervous system damage and recovery. J Neurosci Methods 227:166-180, 2014

57. Senggunprai L, Kukongviriyapan V, Prawan A, Kukongviriyapan U: Quercetin and EGCG exhibit chemopreventive effects in cholangiocarcinoma cells via suppression of JAK/STAT signaling pathway. Phytother Res 28:841-848, 2014

58. Sergent T, Piront N, Meurice J, Toussaint O, Schneider YJ: Anti-inflammatory effects of dietary phenolic compounds in an in vitro model of inflamed human intestinal epithelium. Chem Biol Interact 188:659-667, 2010

59. Shirai N, Suzuki H: Effect of dietary docosahexaenoic acid and catechins on maze behavior in mice. Ann Nutr Metab 48:51-58, 2004

60. Srividhya R, Jyothilakshmi V, Arulmathi K, Senthilkumaran V, Kalaiselvi P: Attenuation of senescence-induced oxidative exacerbations in aged rat brain by (-)-epigallocatechin-3gallate. Int J Dev Neurosci 26:217-223, 2008

61. Stylianou E, Saklatvala J: Interleukin-1. Int J Biochem Cell Biol 30:1075-1079, 1998

62. Sutherland BA, Rahman RM, Appleton I: Mechanisms of action of green tea catechins, with a focus on ischemiainduced neurodegeneration. J Nutr Biochem 17:291-306, 2006

63. Thalhammer JG, Vladimirova M, Bershadsky B, Strichartz GR: Neurologic evaluation of the rat during sciatic nerve block with lidocaine. Anesthesiology 82:1013-1025, 1995

64. Tiwari V, Kuhad A, Chopra K: Amelioration of functional, biochemical and molecular deficits by epigallocatechin gallate in experimental model of alcoholic neuropathy. Eur $\mathbf{J}$ Pain 15:286-292, 2011

65. Turgut M, Kaplan S, Unal BZ, Bozkurt M, Yürüker S, Yenisey C, et al: Stereological analysis of sciatic nerve in chickens following neonatal pinealectomy: an experimental study. J Brachial Plex Peripher Nerve Inj 5:10-16, 2010

66. Tyurin VA, Tyurina YY, Borisenko GG, Sokolova TV, Ritov VB, Quinn PJ, et al: Oxidative stress following traumatic brain injury in rats: quantitation of biomarkers and detection of free radical intermediates. J Neurochem 75:2178-2189, 2000

67. Unal B, Tan H, Orbak Z, Kiki I, Bilici M, Bilici N, et al: Morphological alterations produced by zinc deficiency in rat sciatic nerve: a histological, electron microscopic, and stereological study. Brain Res 1048:228-234, 2005

68. Unno K, Takabayashi F, Kishido T, Oku N: Suppressive effect of green tea catechins on morphologic and functional regression of the brain in aged mice with accelerated senescence (SAMP10). Exp Gerontol 39:1027-1034, 2004 
69. van Praag H, Lucero MJ, Yeo GW, Stecker K, Heivand N, Zhao C, et al: Plant-derived flavanol (-)epicatechin enhances angiogenesis and retention of spatial memory in mice. $\mathbf{J}$ Neurosci 27:5869-5878, 2007

70. Varejão AS, Cabrita AM, Meek MF, Bulas-Cruz J, MeloPinto P, Raimondo S, et al: Functional and morphological assessment of a standardized rat sciatic nerve crush injury with a non-serrated clamp. J Neurotrauma 21:1652-1670, 2004

71. Varejão AS, Melo-Pinto P, Meek MF, Filipe VM, Bulas-Cruz $\mathrm{J}$ : Methods for the experimental functional assessment of rat sciatic nerve regeneration. Neurol Res 26:186-194, 2004

72. Wang T, Zhou H, Xie H, Mu Y, Xu Y, Liu J, et al: Epigallocatechin-3-gallate inhibits TF and TNF- $\alpha$ expression induced by the anti- $\beta 2 \mathrm{GPI} / \beta 2 \mathrm{GPI}$ complex in human THP-1 cells. Int J Mol Med 33:994-1002, 2014

73. Weinreb O, Amit T, Mandel S, Youdim MBH: Neuroprotective molecular mechanisms of (-)-epigallocatechin-3-gallate: a reflective outcome of its antioxidant, iron chelating and neuritogenic properties. Genes Nutr 4:283-296, 2009

74. Xie J, Jiang L, Zhang T, Jin Y, Yang D, Chen F: Neuroprotective effects of epigallocatechin-3-gallate (EGCG) in optic nerve crush model in rats. Neurosci Lett 479:26-30, 2010
75. Yoo KY, Choi JH, Hwang IK, Lee CH, Lee SO, Han SM, et al: (-)-Epigallocatechin-3-gallate increases cell proliferation and neuroblasts in the subgranular zone of the dentate gyrus in adult mice. Phytother Res 24:1065-1070, 2010

\section{Disclosures}

The authors report no conflict of interest concerning the materials or methods used in this study or the findings specified in this paper.

\section{Author Contributions}

Conception and design: all authors. Acquisition of data: all authors. Analysis and interpretation of data: all authors. Drafting the article: Renno. Critically revising the article: all authors. Reviewed submitted version of manuscript: all authors. Approved the final version of the manuscript on behalf of all authors:

Renno. Statistical analysis: Renno. Administrative/technical/material support: Renno. Study supervision: Renno.

\section{Correspondence}

Waleed M. Renno, Department of Anatomy, Faculty of Medicine, Kuwait University, PO Box 24923, Safat 13110, Kuwait. email: wrenno@hsc.edu.kw. 